\title{
Instrumental and analytic methods for bolometric polarimetry
}

\author{
W. C. Jones ${ }^{1}$, T. E. Montroy ${ }^{2}$, B. P. Crill ${ }^{3}$, C. R. Contaldi ${ }^{4}$, T. S. Kisner ${ }^{2,5}$, A. E. Lange ${ }^{1}$, C. J. MacTavish ${ }^{6}$, \\ C. B. Netterfield ${ }^{7,8}$, and J. E. Ruhl ${ }^{2}$ \\ ${ }^{1}$ Division of Physics, Math, and Astronomy, California Institute of Technology, USA \\ e-mail: wcj@astro.caltech.edu \\ 2 Physics Department, Case Western Reserve University, USA \\ 3 Infrared Processing and Analysis Center, California Institute of Technology, USA \\ 4 Department of Physics, Imperial College London, UK \\ 5 Department of Physics, University of California, Santa Barbara, USA \\ 6 Canadian Institute for Theoretical Astrophysics, University of Toronto, Canada \\ 7 Department of Physics, University of Toronto, Canada \\ 8 Department of Astronomy and Astrophysics, University of Toronto, Canada
}

Received 26 June 2006 / Accepted 16 May 2007

\begin{abstract}
Aims. We discuss instrumental and analytic methods that have been developed for the first generation of bolometric cosmic microwave background (CMB) polarimeters. The design, characterization, and analysis of data obtained using Polarization Sensitive Bolometers (PSBs) are described in detail. This is followed by a brief study of the effect of various polarization modulation techniques on the recovery of sky polarization from scanning polarimeter data.

Methods. Having been successfully implemented on the sub-orbital BOOMERANG experiment, PSBs are currently operational in two terrestrial CMB polarization experiments (QUaD and the Robinson Telescope). We investigate two approaches to the analysis of data from these experiments, using realistic simulations of time ordered data to illustrate the impact of instrumental effects on the fidelity of the recovered polarization signal.

Results. We find that the analysis of difference time streams takes full advantage of the high degree of common mode rejection afforded by the PSB design. In addition to the observational efforts currently underway, this discussion is directly applicable to the PSBs that constitute the polarized capability of the Planck HFI instrument.
\end{abstract}

Key words. cosmic microwave background - polarization - instrumentation: detectors - instrumentation: polarimeters techniques: polarimetric - methods: numerical

\section{Introduction}

Recent advances in millimeter-wave instrumentation and techniques have transformed observational Cosmic Microwave Background (CMB) research. Over the course of the past decade, statistical detections of the minute temperature variations in the CMB have given way to high signal to noise imaging of the surface of last scattering.

The rapid pace of technological development in the field of CMB research has contributed to an equally remarkable rate of progress in our understanding of the Universe; cosmology is in the midst of an abrupt transition from a data-starved theoretical framework to a rigorously tested standard model. Remarkably, as of early 2006, the currently avaliable CMB data are sufficiently precise (and accurate!) that, within the framework of the most simple inflationary models, the majority of the scientific potential of the CMB temperature anisotropies has already been realized. Measurements of the polarization of the CMB provide an important confirmation of the validity of the theoretical framework through which the data are interpreted, and also aid in the precise determination of the parameters of the theory.

Further motivation comes from the tantalizing possibility that the CMB polarization holds a unique imprint from gravitational waves generated during the epoch of Inflation. A detection of this signature would represent a probe of physics beyond the standard model - both that of cosmology, and potentially that of particle physics.

Current experimental efforts are focused on the development of high fidelity CMB polarimeters capable of characterizing the small fractional polarization of the CMB. The BOOMERANG03 experiment was the first bolometric instrument to measure the polarization in the CMB, and the first of several to use the Polarization Sensitive Bolometers (PSBs) developed for Planck HFI. Two additional terrestrial telescopes using PSBs, Quad and the Robinson Telescope, are currently observing from the South Pole.

We describe the experimental approach employed by the first generation of bolometric instruments to successfully probe CMB polarization and discuss aspects of the data analysis which may inform future observations. Following a brief description of PSBs, we provide a pedagogical description of the method of analysis that has been applied to the B03 data (Masi et al. 2005; Jones et al. 2006; Piacentini et al. 2006; Montroy et al. 2006; MacTavish et al. 2005). Finally, we investigate the merits of several modulation schemes for scanning polarimeters which are directly applicable to current and proposed CMB polarization experiments at the South Pole and from Antarctic Long Duration Balloon flights. 


\section{Polarization sensitive bolometers}

Quasi-total power and correlation receivers (both heterodyne Barkats et al. 2005 and homodyne Jarosik et al. 2003) using low noise front-end amplifier blocks based on HEMT amplifiers are mature technologies at millimeter wavelengths. The fundamental design principles of these receivers are well established and have been used to construct polarimeters at radio to mm-wave frequencies for many years (Spiga et al. 2002; O'Dell et al. 2003; Barkats et al. 2004; Leitch et al. 2002; Readhead et al. 2004). Although cryogenic bolometric receivers achieve much higher instantaneous sensitivities over wider bandwidths than their coherent analogs, the intrinsic polarization sensitivity of coherent systems has made them the technology of choice for the first generation of CMB polarization experiments.

Prior to the release of the BOOMERANG03 results (Montroy et al. 2006; Piacentini et al. 2006; Jones et al. 2006), all published detections of CMB polarization were obtained from experiments relying on the proven HEMT technology (Leitch et al. 2004; Readhead et al. 2004; Barkats et al. 2004; Hinshaw et al. 2003; Kogut et al. 2003; Page et al. 2003). While interferometry is a robust method of polarimetry, the $N^{2}$ scaling of the complexity of correlators prohibits scaling of the design to large optical throughput, limiting the raw sensitivity of a practical interferometric experiment. Although much progress has been made in increasing the sensitivity, and decreasing the footprint, of singledish HEMT based correlation receivers (Gaier et al. 2003), this technology has not yet been demonstrated with the sensitivity or scalability of contemporary low-background bolometer arrays at frequencies above $\sim 90 \mathrm{GHz}$.

We briefly describe a bolometric system that combines the sensitivity, stability, and scalability of a cryogenic bolometer with the intrinsic polarization capability traditionally associated with coherent systems (Jones et al. 2003). In addition, the design obviates the need for orthogonal mode transducers (OMTs), hybrid tee networks, waveguide plumbing, or quasi-optical beam splitters whose size and weight make fabrication of large format arrays impractical. Polarization sensitive bolometers (PSBs) are fabricated using the proven photolithographic techniques used to produce "spider web" bolometers, and enjoy the same benefits of reduced heat capacity, negligible cross section to cosmic rays, and structural rigidity (Yun 2003). Finally, unlike OMTs or other waveguide devices, these systems can be relatively easily scaled to $\sim 600 \mathrm{GHz}$, limited at high frequencies only by the ability to reliably manufacture sufficiently small single-moded corrugated structures. Receivers using this design have been demonstrated at 100, 150, 217 and $353 \mathrm{GHz}$.

Polarization sensitivity is achieved by controlling the vector surface current distribution on the absorber, and thus the efficiency of the ohmic dissipation of incident Poynting flux. This approach requires that the optics, filtering, and coupling structure preserve the sense of polarization of the incident radiation with high fidelity. A multi-stage corrugated feed structure and coupling cavity has been designed that achieves polarization sensitivity over a $33 \%$ bandwidth.

The PSB design has been driven by the desire to minimize systematic contributions to the polarized signal. Both senses of linearly polarized radiation propagate through a single optical path and filter stack prior to detection, thereby assuring both detectors have identical spectral passbands and closely matched quantum efficiencies.

Two orthogonal free-standing lossy grids, separated by $\sim 60 \mu \mathrm{m}$ and both thermally and electrically isolated, are impedance-matched to terminate a corrugated waveguide structure. The physical proximity of the two detectors assures that both devices operate in identical RF and thermal environments. A printed circuit board attached to the module accommodates load resistors and RF filtering on the leads entering the bolometer cavity. For BoOMERANG, Planck HFI, QUaD, and the Robinson Telescope, the post-detection electronics consist of a highly stable AC readout with a system $1 / f$ knee below $30 \mathrm{mHz}$ (Lamarre et al. 2003; Bowden et al. 2004; Yoon et al. 2006). Unlike coherent systems, this low frequency stability is attained without phase switching the RF signal.

We have designed the optical elements, including the feed antenna and detector assembly, to preserve sky polarization and minimize instrumental polarization of unpolarized light. To this end, the detector has been designed as an integral part of the optical feed structure. Corrugated feeds couple radiation from the telescope to the detector assembly. Corrugated horns are the favored feed element for high performance polarized reflector systems due to their superior beam symmetry, large bandwidth, and low sidelobe levels. In addition, cylindrical corrugated feeds and waveguides preserve the orientation of polarized fields with higher fidelity than do their smooth-walled counterparts.

The coupling structure, which is cooled to below $1 \mathrm{~K}^{1}$, consists of a profiled corrugated horn, a modal filter, and an impedance-matching section that allows efficient coupling to the polarization sensitive bolometer (see Fig. 1). In addition to a reduction in the physical length of the structure, the profiled horn provides a nearly uniform phase front that couples well to the other filters and optical elements in the system. The modal filter isolates the detectors from any unwanted higher order modes that may be excited in the thermal break. In addition, this filter completely separates the design of the bolometer cavity from that of the feed, which couples to the optics. The impedance-matching section (the re-expansion at the left side of Fig. 1) produces a uniform vector field distribution with a well-defined guide wavelength ${ }^{2}$ and characteristic impedance over a large $(\sim 33 \%)$ bandwidth.

The detector assembly is a corrugated waveguide that is operated well above cutoff. The bolometers, which act as an impedance-matched termination of the waveguide cavity, are coupled via a weak thermal link to the temperature bath. The electric field in the cavity drives currents on the surface of the absorber, resulting in ohmic power dissipation in the bolometer. This power is detected as a temperature rise measured by means of matched Neutron Transmutation Doped Germanium (NTDGe) thermistors (Beeman 2001). The bolometers each couple to a single (mutually orthogonal) linear polarization by precisely matching the absorber geometry to the vector field of the coupling structure. The coupling structure has been tailored to ensure that the field distribution resulting from a polarized source is highly linear at the location of the bolometer.

Because the absorber geometry influences the field distribution within the coupling structure, a treatment of the bolometer cavity as a black-body is in general not valid. An important consequence of this fact is that any attempt to model an analogous few-moded ${ }^{3}$ optical system must consider interference terms between modes when calculating coupling efficiencies or simply

${ }^{1}$ The PSB feeds in BoOMERANG03, Quad, and the Robinson Telescope operate at $240 \mathrm{mK}$, while the Planck HFI focal plane is cooled to $\sim 100 \mathrm{mK}$.

2 The guide wavelength, $\lambda_{\mathrm{g}}$, is typically $20 \%$ larger than free space, and $\mathrm{d} \log \left(\lambda_{\mathrm{g}}\right) / \mathrm{d} \log (v)$ remains small over the entire range of operation.

3 By "few-moded" we mean a system with optical throughput, $1<$ $A \Omega / \lambda^{2} \lesssim 10$. 


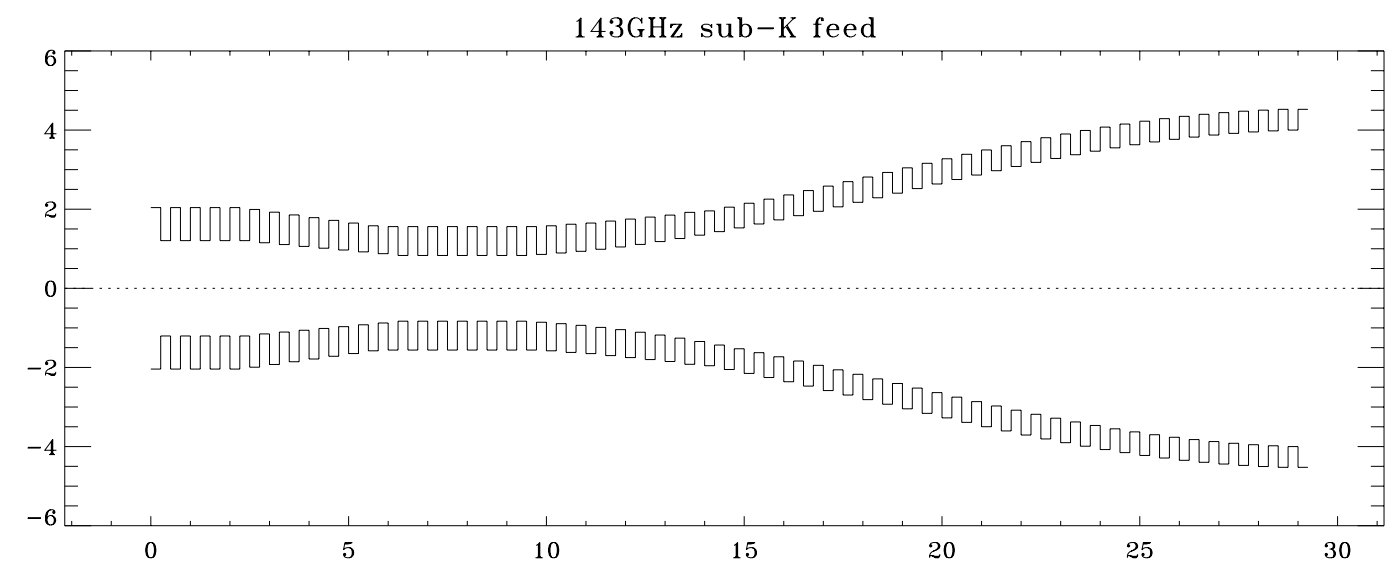

Fig. 1. The corrugation geometry of the BoOMERANG PSB feeds, with dimensions in millimeters. The radiating aperture is on the right hand side, while the PSB module is seated on the left.

trying to predict radiation patterns. The amplitude and phase of any higher order modes capable of propagating to the bolometer depend on the details of both the excitation and structure. Therefore, any numerical calculation would be susceptible to a large number of uncertainties associated with the appropriate boundary conditions at the bolometer. For this reason, it may prove difficult to extend the general single mode PSB design to a few-moded application without sacrificing crosspolar performance.

\section{Analysis}

\subsection{Polarization formalisms}

The two most commonly used conventions for treating polarized radiation are the Jones and the Stokes/Mueller formalisms. The primary difference between the two approaches is that the Stokes/Mueller formalism manipulates irradiances, and therefore is applicable only to incoherent radiation. On the other hand, the Jones formalism models optical elements with matrix operations on the (complex) field amplitudes, making it the appropriate approach for coherent analysis. While the Jones formalism is rather intuitive, the Stokes formalism is more naturally suited to CMB analysis. In the following we introduce both approaches at an elementary level, and describe the correspondence between the two. A more detailed description of each approach may be found in Mueller (1948); Jones (1941a,b, 1942); Hecht (1998) and Hamaker \& Bregman (1996).

The general action of linear optical elements can be described in terms of the relationship between the input and output electric field vectors. The Jones matrix of an optical element is defined in terms of its action on the incident fields,

$\mathbf{e}_{f}=\mathbf{J} \mathbf{e}_{i}$,

where the Jones matrix, $\mathbf{J}$, of the system is a general product of the matrices describing individual components in the system.

$\left(\begin{array}{l}E_{x} \\ E_{y}\end{array}\right)_{f}=\left[\begin{array}{ll}J_{x x} & J_{x y} \\ J_{y x} & J_{y y}\end{array}\right]_{0} \cdots\left[\begin{array}{ll}J_{x x} & J_{x y} \\ J_{y x} & J_{y y}\end{array}\right]_{n}\left(\begin{array}{l}E_{x} \\ E_{y}\end{array}\right)_{i}$.

Of course, all such components may be rotated with respect to one another with the usual rotation matrices,

$\mathbf{J}^{\prime}=\mathbf{R} \mathbf{J} \mathbf{R}^{T}$,

with

$R \equiv\left(\begin{array}{cc}\cos \psi & -\sin \psi \\ \sin \psi & \cos \psi\end{array}\right)$

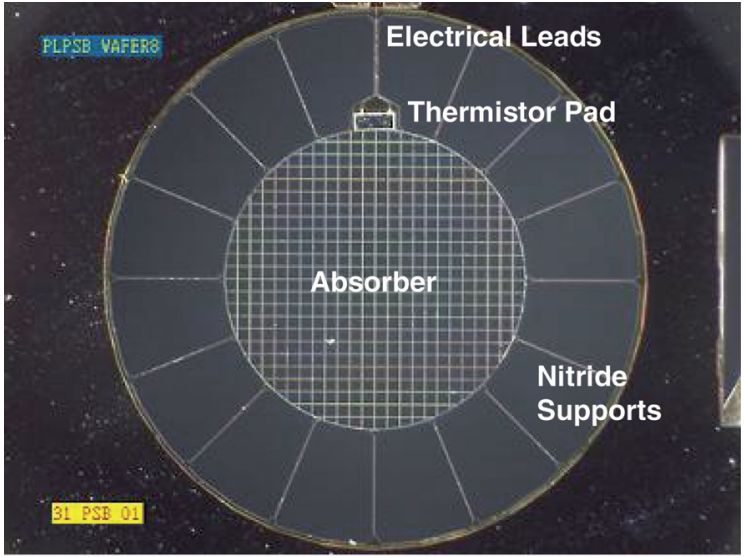

Fig. 2. A photograph of a $145 \mathrm{GHz}$ BoomerANG PSB absorber. The diameter of the grid is $2.6 \mathrm{~mm}$, while the absorber leg spacing, $g$, is $108 \mu \mathrm{m}$. Each leg is $3 \mu \mathrm{m}$ wide. This device is sensitive to incident radiation polarized in the vertical direction due to the metalization of the $\mathrm{Si}_{3} \mathrm{~N}_{4}$ mesh in that direction. The horizontal $\mathrm{Si}_{3} \mathrm{~N}_{4}$ beams evident in the photo are not metalized, and provide structural support for the device. The thermal conductivity between the absorber and the heat sink is dominated by the metallic leads running to the thermistor chip.

This formalism allows a fairly complicated optical system to be described by a single matrix, which need only be derived once from the constituent components.

As an example we describe an imperfect polarizer oriented at an angle $\psi$ with respect to the basis in which the fields are defined. Such an object may be represented by the Jones matrix:

$$
\begin{aligned}
\mathbf{J}_{p} & \equiv \mathbf{R}\left[\begin{array}{ll}
\eta & 0 \\
0 & \delta
\end{array}\right] \mathbf{R}^{T} \\
& =\left[\begin{array}{cc}
\eta \cos ^{2} \psi+\delta \sin ^{2} \psi & (\eta-\delta) \cos \psi \sin \psi \\
(\eta-\delta) \cos \psi \sin \psi & \eta \sin ^{2} \psi+\delta \cos ^{2} \psi
\end{array}\right],
\end{aligned}
$$

where $\eta>\delta$. A perfect polarizer would have $\eta=1$ and $\delta=0$. After generous application of trigonometric identities, one recovers the general Jones matrix for an imperfect polarizer oriented at an angle $\psi$

$\mathrm{J}_{p}=\frac{1}{2}\left[\begin{array}{cc}(\eta+\delta)+(\eta-\delta) \cos 2 \psi & (\eta-\delta) \sin 2 \psi \\ (\eta-\delta) \sin 2 \psi & (\eta+\delta)-(\eta-\delta) \cos 2 \psi\end{array}\right]$.

Each detector in a polarization sensitive bolometer pair acts as just such a partial polarizer, followed by a total power detector. 


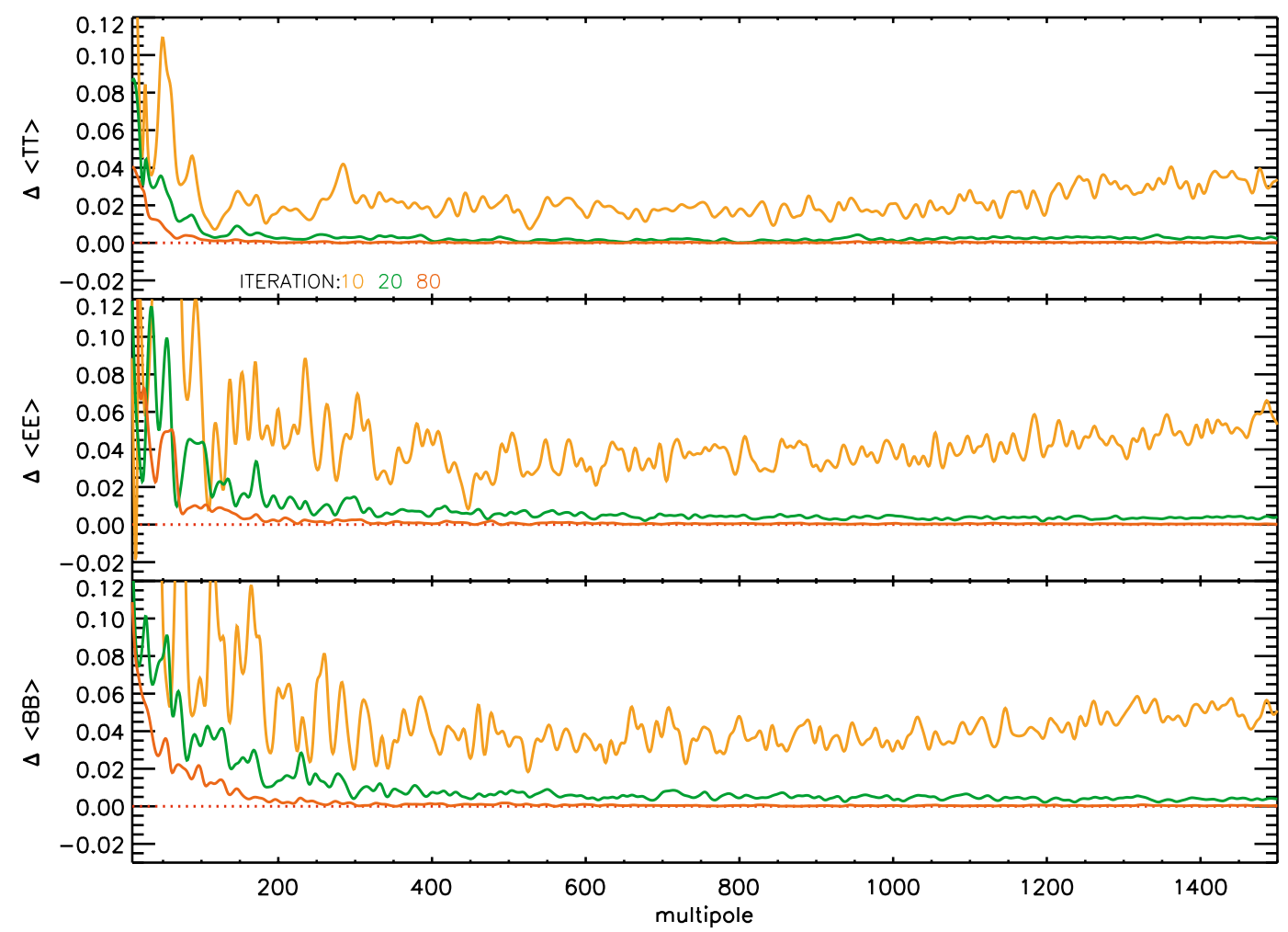

Fig. 3. The fractional change in the cut-sky angular power spectra (pseudo- $C_{\ell}$ ) for temperature (top panel), and polarization (bottom panels) from iteration to iteration of the mapmaker. The largest scales take the longest to converge, and the polarization signal generally takes longer to converge than does the temperature.
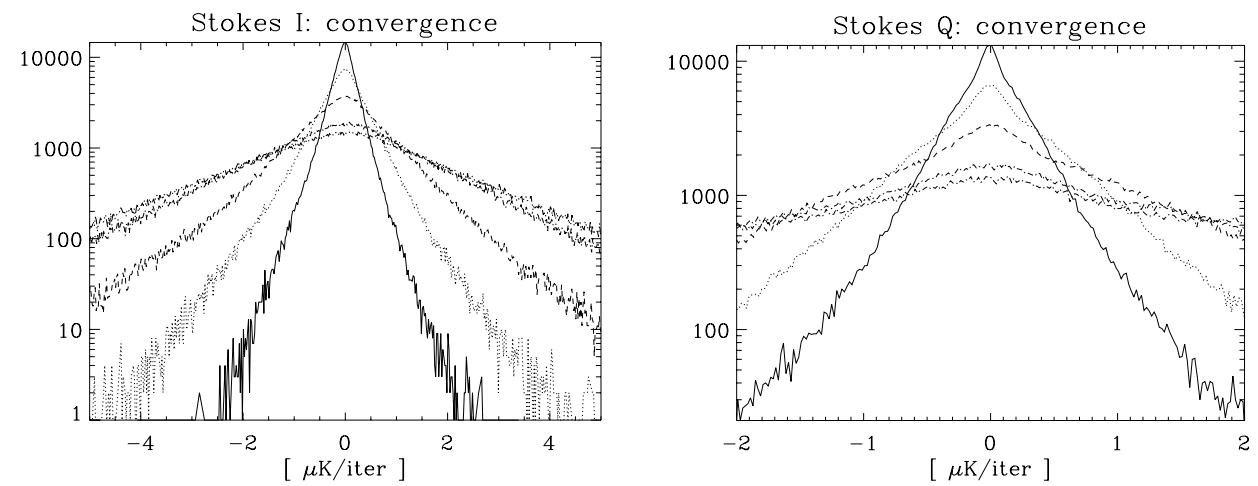

Fig. 4. The convergence criteria for the iterative procedure is determined by a threshold on the rms amplitude of the correction. Histograms of the corrections to the $I$ (left panel) and $Q$ (right panel) maps (the change in pixel value between subsequent iterations) are shown for 5, 10, 20, 40 and 80 iterations. For comparison, the noise per pixel of the B03 temperature data at the same resolution (3.4') is typically $\sim 24 \mu \mathrm{K}$ (Jones et al. 2006).

The Stokes parameters are defined in terms of the electric field as follows:

$$
\begin{aligned}
& I \equiv\left\langle E_{x} E_{x}^{*}+E_{y} E_{y}^{*}\right\rangle=\left\langle\left|E_{x}\right|^{2}\right\rangle+\left\langle\left|E_{y}\right|^{2}\right\rangle \\
& Q \equiv\left\langle E_{x} E_{x}^{*}-E_{y} E_{y}^{*}\right\rangle=\left\langle\left|E_{x}\right|^{2}\right\rangle-\left\langle\left|E_{y}\right|^{2}\right\rangle \\
& U \equiv\left\langle E_{x} E_{y}^{*}+E_{y} E_{x}^{*}\right\rangle=2\left\langle\left|E_{x} E_{y}\right| \cos \left(\phi_{x}-\phi_{y}\right)\right\rangle \\
& V \equiv i\left\langle E_{x} E_{y}^{*}-E_{y} E_{x}^{*}\right\rangle=2\left\langle\left|E_{x} E_{y}\right| \sin \left(\phi_{x}-\phi_{y}\right)\right\rangle
\end{aligned}
$$

where the brackets, \langle\rangle , represent a time average and the fields are specified in a coordinate system fixed with respect to the instrument. For Thomson scattering of electrons in a quadrupolar radiation field there is no mechanism for the introduction of a relative phase between the two polarizations. Therefore, the cosmological Stokes $V$ parameter is presumed to be zero.

The action of linear optical elements on a Stokes vector, $\mathbf{s}$, can be described in terms of the elements' Mueller matrix, $\mathbf{s}_{f}=\mathbf{M} \mathbf{s}_{i}$
Given the definition of the Stokes parameters, one can derive the relationship between a Jones matrix, and the corresponding Mueller matrix. Following Born \& Wolf (1980) we find

$M_{i j}=\frac{1}{2} \operatorname{tr}\left(\sigma_{\mathbf{i}} \mathbf{J} \sigma_{\mathbf{j}} \mathbf{J}^{\dagger}\right)$,

where the $\sigma_{i}$ are the Pauli matrices:

$$
\begin{gathered}
\sigma_{I}=\sigma_{0} \equiv\left(\begin{array}{ll}
1 & 0 \\
0 & 1
\end{array}\right) \sigma_{Q}=\sigma_{3} \equiv\left(\begin{array}{cc}
1 & 0 \\
0 & -1
\end{array}\right) \\
\sigma_{U}=\sigma_{1} \equiv\left(\begin{array}{ll}
0 & 1 \\
1 & 0
\end{array}\right) \sigma_{V}=\sigma_{2} \equiv\left(\begin{array}{cc}
0 & -i \\
i & 0
\end{array}\right) .
\end{gathered}
$$

Applying a moderate amount of algebra to Eqs. (4) and (5), we find the first row of the Mueller matrix $\mathbf{M}_{p}$ for a partial polarizer. This defines the total power detected as a function of the 


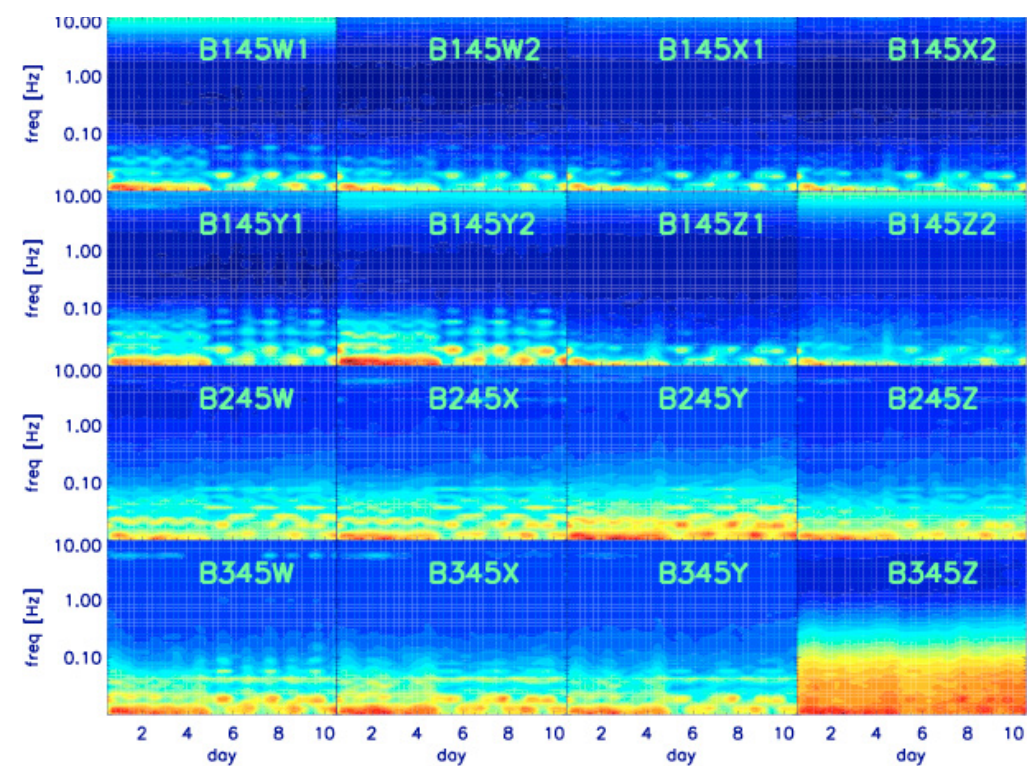

Fig. 5. The time dependence of the (signal subtracted) noise power spectra of the BooMERANG science channels as determined from the in-flight data. Each frame shows the power spectrum of each noise stationary subset (chunk) from a particular channel. The series of lines above $10 \mathrm{mHz}$ corresponds to the harmonics of the scan frequency. The signal band extends from 0.05 to $5 \mathrm{~Hz}$. The diurnal dependence of the $1 / f$ knee is evident. The B345Z channel exhibited noise whose properties were neither stationary nor Gaussian, which is manifest in the low frequency contribution.

incident $I, Q, U$, and $V$ parameters:

$$
\begin{aligned}
M_{I I} & =\frac{1}{2}\left(\eta^{2}+\delta^{2}\right) \\
M_{I Q} & =\frac{1}{2}\left(\eta^{2}-\delta^{2}\right) \cos 2 \psi \\
M_{I U} & =\frac{1}{2}\left(\eta^{2}-\delta^{2}\right) \sin 2 \psi \\
M_{I V} & =0 .
\end{aligned}
$$

The signal from a total power detector is proportional to the Stokes $I$ parameter of the incident radiation. Modeling a polarization sensitive bolometer as a partial polarizer followed by a total power detector, we find (ignoring, for the moment, the effects of finite beam size and frequency passband) the data may be expressed as a sum

$$
d_{i}=\frac{s}{2}\left[(1+\epsilon) \cdot I+(1-\epsilon) \cdot\left(Q \cos 2 \psi_{i}+U \sin 2 \psi_{i}\right)\right]+n_{i},
$$

where we have defined the polarization leakage term, $\epsilon$, such that $(1-\epsilon)$ is the polarization efficiency ${ }^{4}, \psi$ is the orientation of the axis of sensitivity of the PSB, and $s$ is the voltage responsivity of the detector. For BoOMERANG, the value of the crosspolar leakage is typically $\sim 5 \%$, and ranges from $2-7 \%$ for second generation devices designed for the Planck HFI, BICEP, and QUAD.

It should be noted that the noise contribution, $n$, is overly simplified in Eq. (11). See Appendix A for a more detailed discussion of the noise properties of bolometric receivers, which explain the general features of the noise power spectra shown in Figs. 5 and 6.

4 That is, in terms of the elements of the Jones matrix for an imperfect polarizer, the leakage $\epsilon \equiv \delta^{2} / \eta^{2}$. This is the ratio between the minimum and peak power response to a pure linearly polarized source, which is a directly observable property of the PSB.

\subsection{Polarized beams}

The angular response of an instrument can be characterized by the copolar and crosspolar power response functions $P_{\|}(r, \theta, \phi)$ and $P_{\perp}(r, \theta, \phi)$. In the time reversed sense these can be thought of as the normalized power at any point in space resulting from a linearly polarized excitation produced by the feed element in the focal plane. That is, for a given polarization $p=\{\|, \perp\}$,

$P_{p}(r, \theta, \phi) \equiv \frac{\left|E_{p}(r, \theta, \phi)\right|^{2}}{\left|E_{\|}(r, 0,0)\right|^{2}}$.

For a single moded system, $P_{p}$ has nothing to do with the properties of the detector. Due to the presence of the modal filter in the throat of the coupling feed (see Fig. 1), the beam is a function only of the feed geometry and the optical elements of the system. To fully characterize the system, the polarized beam patterns must be considered separately from the detector.

The exact definitions used for the polarizations on the sphere vary in the literature, but the standard is Ludwig's Third definition Ludwig (1973). In any case, for small angles from the beam centroid, they are very nearly equal to the Cartesian definition.

The copolar beam, $P_{\|}$, is qualitatively similar to an Airy pattern; a Gaussian near the beam centroid, with a series of sidelobes. For most optical systems the crosspolar beam, $P_{\perp}$, reaches a minimum at the peak of the copolar beam and, for on-axis systems, is minimized along both the E- and H-planes. The peak of the crosspolar pattern typically occurs near the half-power point of the copolar beam, and the peak amplitude relative to the copolar beam is fundamentally related to the asymmetry of the copolar beam (Olver et al. 1994). For an azimuthally symmetric system, such as a feedhorn antenna, this produces lobes in the $45^{\circ}$ plane in all four quadrants of the beam. For an offaxis reflector such as the BOOMERANG telescope, the azimuthal symmetry is lost and the lobes are bimodal. It is worth noting that the polarized beams generally depend on frequency as well as the field distance.

A PSB detects the convolution of the polarized sky with the polarized beam, integrated over the frequency bandpass, and 

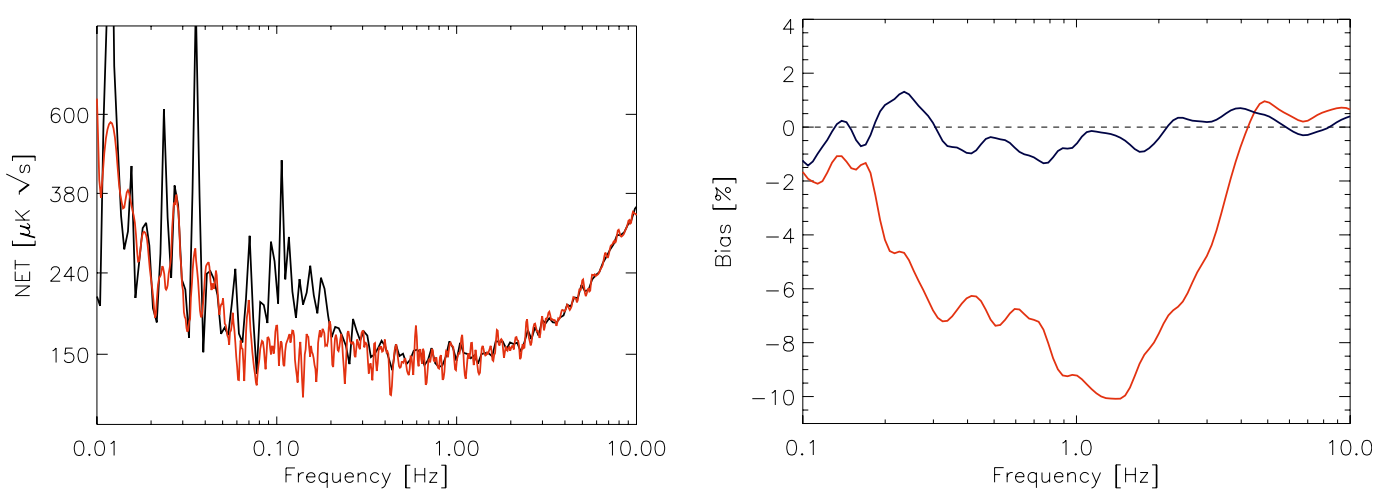

Fig. 6. Left panel: the power spectral density, in CMB units, of the noise for a representative chunk of the deconvolved B03 time ordered data. The black line is derived from the raw (signal plus noise) data, whereas the red line is the estimate of the signal-subtracted PSD. The scan frequency for this chunk appears at $12 \mathrm{mHz}$, and the CMB dipole (which appears as a triangle wave at the scan frequency) has been subtracted from the TOD prior to the noise estimation. Right panel: the amplitude of the noise bias as determined from an ensemble of signal plus noise simulations. The blue line is representative of the bias in a typical high signal-to-noise chunk, whereas the red line is the most extreme example found in the low signal-to-noise regime. Further discussion of the noise in bolometric detectors can be found in Appendix A.

subject to the polarization efficiency of the detector ${ }^{5}$. In the flat sky approximation, a time domain sample of a single detector within a PSB pair, $d_{i}$, may therefore be written as the sum of a signal component

$$
\begin{aligned}
& d_{i}=\frac{s}{2} \int \mathrm{d} v \frac{\lambda^{2}}{\Omega_{\mathrm{b}}} F_{v} \iint \mathrm{d} \Omega\left(P_{\|}\left(\hat{r}_{i}\right)+P_{\perp}\left(\hat{r}_{i}\right)\right)\left[I+\gamma \mathcal{P}\left(\hat{r}_{i}\right)\right. \\
&\left.\times\left(Q \cos 2 \psi_{i}+U \sin 2 \psi_{i}\right)\right],
\end{aligned}
$$

and a noise contribution. Here, the Stokes parameters are defined on the full sky and the integration variable is $\hat{r}_{i}=\hat{n}_{i}-\hat{r}$, for a vector, $\hat{n}_{i}$, describing the pointing at a time sample, $i$. We have also defined the beam solid angle $\Omega_{\mathrm{b}}=\iint \mathrm{d} \Omega\left(P_{\|}+P_{\perp}\right)$. The normalized beam response and the polarization efficiency are given by,

$\mathcal{P}(\hat{r}) \equiv \frac{P_{\|}-P_{\perp}}{P_{\|}+P_{\perp}} \quad \gamma \equiv \frac{1-\epsilon}{1+\epsilon}$.

For $\mathrm{B} 03$, the angle $\psi$ is modulated by sky rotation and the motion of the gondola. The calibration factor, $s$, converts the brightness fluctuations in $I, Q$, and $U$ to a signal voltage.

By rearranging Eq. (13) and dropping both the explicit spatial and frequency dependencies, the relation can be written more intuitively,

$d_{i} \simeq \frac{s}{2} \int \mathrm{d} v \lambda^{2} F_{v} \iint \mathrm{d} \Omega\left[I+\gamma \mathcal{P}\left(Q \cos 2 \psi_{i}+U \sin 2 \psi_{i}\right)\right]$.

We have made the simplifying assumption that we may remove the beam and polarization efficiencies from the integral over the sky, and then absorb these prefactors into a redefinition of the calibration constant, $s=s^{\prime} \int \mathrm{d} v(1+\epsilon)$.

5 This treatment is actually more general; it holds for any receiver that can be characterized as a total power detector preceded by an imperfect polarizer. That is, any receiver that is well described by a Jones matrix of the type

$J_{p}=\left[\begin{array}{ll}\eta & 0 \\ 0 & \delta\end{array}\right]$.

In the discussion that follows, keep in mind that $\epsilon \equiv \frac{\delta^{2}}{\eta^{2}}$.

\subsection{Signal and noise estimation}

A great deal of effort has been devoted to the development of algorithms designed to estimate the signal and noise from noisedominated data, and a rich literature has developed around the topic (for some recent examples, see Jarosik et al. 2006; Doré et al. 2001; Amblard \& Hamilton 2004). In this section we outline in pedagogical detail the method used to estimate the signal and noise from the published BoOMERANG03 data (MacTavish et al. 2005; Montroy et al. 2006; Piacentini et al. 2006; Jones et al. 2006).

An estimate of the instrumental noise properties that is both precise and accurate is required in order to avoid the introduction of a bias to the estimate of the power spectrum of the signal. For B03, the high signal to noise ratio of the data in fact complicates the noise estimation procedure. We solve for the noise and signal simultaneously using an iterative procedure adapted from that applied in the analysis of the data from the 1998 flight of BOOMERANG (Prunet et al. 2001; Netterfield et al. 2002; Ruhl et al. 2003). The B03 data are unique among contemporary CMB experiments in that not only are the temperature maps signal dominated at angular scales approaching the beamsize, but the time ordered data are also characterized by signal to noise ratios of order unity. B03 is the only polarized dataset that is comparable in this regard to that anticipated from the Planck HFI.

Assuming that the data, $\mathbf{d}$, are well described as the sum of a sky signal and a noise contribution, $\mathbf{d}=\mathbf{A} \mathbf{m}+\mathbf{n}$, where $\mathbf{A}^{T}$ is the pointing matrix which maps time domain samples to pixels on the sky. If the statistical properties of the noise contribution are piecewise stationary with a (circulant) noise covariance matrix, $\mathbf{N}$, defined as

$N_{t t^{\prime}}=\frac{1}{N} \sum_{i}^{N_{d}}\left(n_{i+t}-\langle\mathbf{n}\rangle\right)\left(n_{i+t^{\prime}}-\langle\mathbf{n}\rangle\right)$

then the least squares estimate of the map is given by,

$\widetilde{\mathbf{m}}=\left(\mathbf{A}^{T} \mathbf{N}^{-1} \mathbf{A}\right)^{-1} \mathbf{A}^{T} \mathbf{N}^{-1} \mathbf{d}$.

The iterative procedure begins with the assumption of a white noise power spectrum (i.e., diagonal $\mathbf{N}$ ), in which case Eq. (16) corresponds to a simple average of the data falling in a given pixel. The noise contribution used in a given iteration on the 
solution to Eq. (16) is obtained from the estimate of the signal obtained in the previous iteration,

$\widetilde{\mathbf{n}}_{k+1}=\mathbf{d}-\mathbf{A} \widetilde{\mathbf{m}}_{k}$.

Piecewise stationarity of the noise allows subsequent convolutions of $\mathbf{N}^{-1} \mathbf{d}$ to be performed in the Fourier domain.

For most terrestrial telescopes, which suffer from relatively high backgrounds and large atmospheric signals, the time stream is dominated by noise. Therefore, a good approximation to the noise covariance matrix appearing in Eq. (16) is provided by the power spectrum of the raw data. For orbital and balloon-based CMB experiments like BOOMERANG, the time ordered data are not noise dominated, greatly complicating an accurate determination of the noise. In general, an in-situ estimation of the noise is required due to the influence of atmospheric emission, unpredictable backgrounds, and scan-synchronous effects. As a result, the simultaneous estimation of both the signal and noise is required.

An iterative solution for both $\mathbf{N}$ and $\widetilde{\mathbf{m}}$ is possible by using an adaptation of the Jacobi method. The Jacobi method is an iterative approach to the solution of a general linear system of equations, such as Eq. (16), that does not require the inversion of large matrices. The application to the solution of Eq. (16) is derived in Appendix B. This algorithm is naturally suited to the problem of noise estimation, as the signal subtraction is an integral part of the iterative procedure.

By iterating on the noise covariance matrix, $\mathbf{N}$, as well as the signal, $\widetilde{\mathbf{m}}$, one approaches a general least squares solution for both. This procedure has been used in the noise estimation of previous experiments that probed the CMB temperature anisotropies (Wright et al. 1996; Prunet et al. 2001). In this application, the approach has been extended to a polarized data set.

As described in Appendix B, each subsequent iteration on the solution to Eq. (16), $\widetilde{\mathbf{m}}_{k+1}$, is calculated from the previous solution according to the procedure

$\widetilde{\mathbf{m}}_{k+1}=\widetilde{\mathbf{m}}_{k}+\delta \widetilde{\mathbf{m}}_{k+1}$,

where

$\delta \widetilde{\mathbf{m}}_{k+1} \equiv \alpha \cdot \operatorname{diag}\left(\mathbf{A}^{T} \mathbf{N}_{k}^{-1} \mathbf{A}\right)^{-1} \mathbf{A}^{T} \mathbf{N}_{k}^{-1}\left(\mathbf{d}-\mathbf{A} \widetilde{\mathbf{m}}_{k}\right)$,

and the relaxation parameter, $\alpha \lesssim 1$, is tuned to optimize the speed of convergence. Recall that, in the case of polarized data, the quantity d represents the left hand side of Eq. (21). Therefore, the calculation of the matrix $\operatorname{diag}\left(\mathbf{A}^{T} \mathbf{N}_{k}^{-1} \mathbf{A}\right)^{-1}$ involves the inversion of the polarization decorrelation matrix on the right hand side of Eq. (21). The great advantage of this method is that the convolution of the data with the inverse noise correlation matrix,

$\mathbf{n}_{k+1} \equiv \mathbf{N}_{k}^{-1}\left(\mathbf{d}-\mathbf{A} \widetilde{\mathbf{m}}_{k}\right)$,

can be efficiently calculated in the Fourier domain, without needing to invert the full time domain correlation matrix, $\mathbf{N}$. This operation is simply the application of a Fourier filter to the signalsubtracted time stream using the inverse of the noise power spectrum as the filter kernel.

The unbiased estimation of power spectra relies crucially on the ability to accurately model the noise properties of the instrument (Hivon et al. 2002; Borrill 1999). In order to treat the noise in a self-consistent fashion as the realization of a Gaussian random process, it is necessary to measure and store the observed auto- and cross-correlations for all channel permutations, and each noise stationary subset. In the North American analysis of
B $03^{6}$, the data are divided into 215 noise-stationary subsets, referred to as chunks, each of which consist of approximately one hour of data. For each of these chunks the 36 (complex) autoand cross-power spectra are calculated, binned logarithmically, and stored to disk. When used to generate noise realizations or construct filtering kernels, these binned spectra are interpolated to the discrete frequencies required by each subset of the data.

The BOOMERANG readout electronics are AC coupled at $\sim 6 \mathrm{mHz}$, and therefore there is no useful information in the time stream on timescales longer than that set by the stationarity of the noise. Dividing the data into these hour long subsets represents a tradeoff between sample variance and stationarity in the accuracy of the noise estimate. The non-stationarity of the B03 noise is illustrated in Fig. 5.

The chunk boundaries are chosen to maximize the accuracy of the noise estimate. The length of these chunks introduces a practical limit to the length, $N_{\tau}$, of the kernel applied in Eq. (18). The computational scaling is thus $N_{d} \log \left(N_{\tau}\right)$ for each iteration of the mapmaker. The memory requirement is also set by the degree of noise stationarity; the algorithm only requires the pointing and bolometer data for an individual chunk to be held in memory at any given time. For B03, the contributions of file writing and Fourier transforms to the run time are approximately equal, depending on the avaliable memory ${ }^{7}$.

The Fourier approach to the analysis requires the data within a chunk to be continuous and well characterized by a given noise power spectrum. About $7 \%$ of the B03 time stream is contaminated by transient events (primarily cosmic ray hits and calibration lamp pulses). These gaps are flagged, and replaced with fake data that are statistically consistent with the remainder of the chunk. The signal subtracted data are easily filled with any reasonable realization of the noise. Due to the small fraction of the data which are contaminated, the exact method of gap-filling has negligible impact on the final signal and noise estimates.

For each chunk all $\left[N_{\mathrm{ch}}\left(N_{\mathrm{ch}}-1\right) / 2+N_{\mathrm{ch}}\right]$ auto and cross power spectra are derived from the signal-subtracted time stream, n, obtained from Eq. (16) using the maximum likelihood maps derived from the full set of data. The noise spectra obtained in this manner are generally biased due to the effect of pixelizing the (continuous) sky signal, as well as the finite signal to noise with which the sky signal, $\widetilde{\mathbf{m}}$, is recovered (Amblard \& Hamilton 2004).

Given a sufficiently high resolution pixelization, the signal variation within a pixel can be made to be negligibly small compared to the noise in the map. We pixelize the sky using the HEALPix method, at a resolution which corresponds to a pixel size of $\simeq 3.4^{\prime}$ (Górski et al. 2005). At this resolution we find the effect of pixelization to be well below the instrumental noise per pixel of the B03 data. As described in Masi et al. (2005) and Jones et al. (2006), the B03 CMB data are divided into a shallow and deep field, the latter being a subset of the former. The noise per pixel of the deep field is roughly three times lower than the shallow field.

The impact of the noise in the signal estimate $i$ found to be significant for the data that constitute the shallow region of the

\footnotetext{
${ }^{6}$ The BoOMERANG team implemented two independent analysis of the time ordered data from the 2003 Antarctic Long Duration Balloon flight. The results from both pipelines are reported in Jones et al. (2006); Montroy et al. (2006); Piacentini et al. (2006); Masi et al. (2005).

7 The Jacobi solver implemented by the North American BOOMERANG team requires $\sim 120 \mathrm{MB}$ of RAM and produces a converged GLS estimate of the signal and noise at a rate of 10 processor-s/channel/hour of data sampled at $60 \mathrm{~Hz}$, when running on a $2 \mathrm{GHz}$ AMD Athlon64 X2 workstation.
} 
B03 target field. The raw sensitivity of the instrument ultimately determines the signal to noise ratio of the time ordered data and, when combined with the distribution of integration time on the sky, the fidelity of the recovered signal estimate, $\widetilde{\mathbf{m}}$. The error in the signal estimate, $\widetilde{\mathbf{m}}$, introduces a bias to the estimate of the noise power spectrum Amblard \& Hamilton (2004). This bias is generally frequency dependent because of the finite bandwidth of the signal. The bias in the noise estimation varies from chunk to chunk as a result of the variation in signal to noise ratio in different parts of the map.

The origin of this bias can be understood through closer examination of the signal-subtracted time stream, $\widetilde{\mathbf{n}}$, that is obtained from the estimate of the Stokes parameter maps, $\widetilde{\mathbf{m}}$, namely, $\widetilde{\mathbf{n}}=\mathbf{d}-\mathbf{A} \widetilde{\mathbf{m}}$. The data are assumed to consist of the sum of a pure signal and noise, $\mathbf{d}=\mathbf{s}+\mathbf{n}$, giving

$$
\begin{aligned}
\widetilde{\mathbf{n}} & =\mathbf{s}+\mathbf{n}-\mathbf{A} \widetilde{\mathbf{m}} \\
& =\mathbf{n}-\hat{\mathbf{n}}
\end{aligned}
$$

where we have defined the projection of the signal error to the time stream as $\hat{\mathbf{n}} \equiv \mathbf{A}(\widetilde{\mathbf{m}}-\mathbf{m})$. The raw noise power spectrum, $\left\langle\widetilde{\mathbf{n n}}^{\dagger}\right\rangle$, which is estimated from Eq. (19) differs from the true noise power spectrum, $\left\langle\mathbf{n} \mathbf{n}^{\dagger}\right\rangle$, by the factor

$\left(1+\frac{\left\langle\hat{\mathbf{n}} \hat{\mathbf{n}}^{\dagger}\right\rangle}{\left\langle\mathbf{n n}^{\dagger}\right\rangle}-2 \frac{\left\langle\hat{\mathbf{n}} \mathbf{n}^{\dagger}\right\rangle}{\left\langle\mathbf{n n}^{\dagger}\right\rangle}\right)$.

For B03, the projection of the map errors to the time domain is highly correlated with the true time domain noise, and therefore the cross-correlation term dominates in Eq. (20). The raw noise power spectra therefore tend to underestimate the true amplitude of the noise at frequencies within the signal bandwidth. The amplitude of the bias term in Eq. (20) is as high as $10 \%$ for the most poorly covered regions in the shallow field, and is below $1 \%$ for the 175 chunks of the deep field.

To correct for the bias present in the B03 noise estimates, we generate an ensemble of signal and noise simulations using a fiducial noise power spectrum and run the noise estimation procedure on each realization. The transfer function of the noise estimation procedure is then obtained by comparing the ensemble average of the estimated noise power spectra to the input power spectra. The size of the ensemble is determined by the required reduction of the sample variance at the lowest frequencies of interest; we find that the transfer function is characterized at the sub-percent level with seventy-five realizations. This bias transfer function is then used to correct the spectra obtained for each chunk of the time ordered data. A comparison of bias transfer functions that are typical of data in the high and low signal to noise regimes is shown in Fig. 6.

In order to produce noise realizations which accurately reflect the statistical properties of the instrumental noise, we require a framework in which to treat noise correlations between detectors in the time domain. Noise correlations in the data are expected both from fundamental considerations (see Appendix A), as well as from the presence of correlated thermal/optical fluctuations, and crosstalk in the readout electronics. The measured noise from a given channel, $\widetilde{n}^{k}$, is modeled as the sum of an intrinsic (uncorrelated) component, $n^{k}$, and the contributions from the intrinsic noise of the other channels, filtered through a (frequency dependent) crosstalk transfer function $\xi_{i k}$.

$\tilde{n}^{k}=n^{k}+\sum_{i \neq k} \xi_{i k} n^{i}$

where, by definition, the intrinsic noise at each frequency is distributed as an uncorrelated Gaussian distribution,

$\left\langle n^{i} n^{k}\right\rangle \equiv \delta_{i k} P_{i k}$.
The observable quantities

$\left\langle\widetilde{n}^{i} \widetilde{n}^{k}\right\rangle=\widetilde{P}_{i k}$

are the $\left(N_{\mathrm{ch}}\left(N_{\mathrm{ch}}-1\right) / 2+N_{\mathrm{ch}}\right)$ auto- and cross-correlations of the signal-subtracted time streams, which are estimated directly from the time ordered data. After correcting for bias, the $\widetilde{P}_{i k}$ are used to generate realizations of the noise time streams which exhibit the same correlation structure observed in the data. These noise realizations are constructed bin-by-bin in the Fourier domain. For each discrete frequency, we calculate the Cholesky factorization, $\widetilde{\mathbf{H}}(f)$, of the complex (Hermitian positive definite) channel correlation matrix,

$\widetilde{\mathbf{P}}(f)=\widetilde{\mathbf{H}}(f) \widetilde{\mathbf{H}}^{T}(f)$.

Independent realizations of white noise are generated for each channel. Simulated data with the proper correlation structure are obtained by operating on the transform of these realizations with the $N_{\text {ch }} \times N_{\text {ch }}$ matrix $\widetilde{H}_{i k}(f)$ for each frequency bin in a given noise-stationary subset of the data. Once all of the frequency components are calculated, the inverse transform provides a correlated noise time stream for each channel that is used in the Monte Carlo pipeline.

\subsection{Polarized mapmaking}

Estimates of the $I, Q$, and $U$ parameters can be recovered by generating orthogonal linear combinations of the data. For each sample, $i$, of a given detector and a measurement of the projection of the orientation of that detector on the sky, $\psi_{i}$, one can construct the polarization decorrelation matrix defined by,

$\left(\begin{array}{c}d_{i} \\ d_{i} \gamma c_{i} \\ d_{i} \gamma s_{i}\end{array}\right)=\left(\begin{array}{ccc}1 & \gamma c_{i} & \gamma s_{i} \\ \gamma c_{i} & \gamma^{2} c_{i}^{2} & \gamma^{2} s_{i} c_{i} \\ \gamma s_{i} & \gamma^{2} c_{i} s_{i} & \gamma^{2} s_{i}^{2}\end{array}\right)\left(\begin{array}{c}I \\ Q \\ U\end{array}\right)$

where $\gamma \equiv \frac{(1-\epsilon)}{(1+\epsilon)}$ is a parameterization of the polarization efficiency. For simplicity we have abbreviated the trigonometric functions, whose argument is $2 \psi_{i}$.

In the limit that the instrumental noise time stream, $\mathbf{n}$, is stationary, Gaussian, and is well-characterized by a white frequency spectrum, the optimal map is obtained by summing all time samples $d_{i}$ and decorrelation matrix elements falling in a pixel $p$. Assuming that the scan strategy, instrument, or channel combination provides modulation of the angle $\psi$, the matrix is nonsingular and the best estimates for $I, Q$, and $U$ are then obtained by inverting the coadded $(3 \times 3)$ decorrelation matrix at each pixel. This is the polarized analog to a naively coadded temperature map.

The situation becomes markedly more difficult in the presence of noise with nontrivial statistics. The solution that is optimal in the least squares sense is again given by Eq. (16), with the understanding that now the data consist of the linear combinations defined by Eq. (21). We now turn to the problem of finding the solution to Eq. (16) for polarized data, in the presence of noise with unknown statistical properties, using channels of varying sensitivity and polarization efficiency. In this general case, the data are treated in the following way: estimates of the left hand side of the noise only version Eq. (21) and the Stokes decorrelation matrix are generated for each pixel,

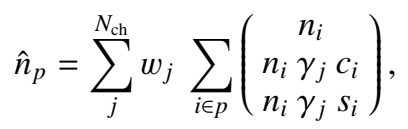




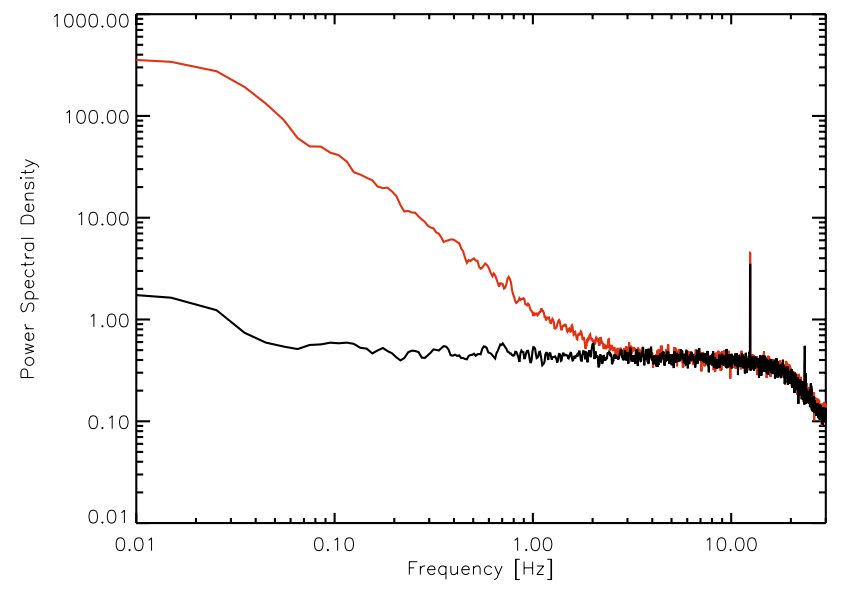

Fig. 7. The power spectrum of sum and difference time streams from a typical $145 \mathrm{GHz}$ PSB pair observing (apparently) unpolarized atmospheric fluctuations during the austral summer in Antarctica. When measuring a small polarized signal buried in a large unpolarized background, the high degree of common mode rejection of the PSBs makes them naturally suited to an analysis of the sum and difference time streams, as described in Sect. 3.5.

where the $n_{i}$ are the elements of signal subtracted time stream. Likewise, for the decorrelation matrix one calculates

$\hat{M}_{p}=\sum_{j}^{N_{\mathrm{ch}}} w_{j} \sum_{i \in p}\left(\begin{array}{ccc}1 & \gamma_{j} c_{i} & \gamma_{j} s_{i} \\ - & \gamma_{j}^{2} c_{i}^{2} & \gamma_{j}^{2} c_{i} s_{i} \\ - & - & \gamma_{j}^{2} s_{i}^{2}\end{array}\right)$.

One then obtains an estimate of the corrections to the Stokes parameters $I, Q$, and $U$ maps for an iteration $k$, by inverting $\hat{M}$ at each pixel,

$\mathbf{S}_{k+1}-\mathbf{S}_{k}=\hat{\mathbf{M}}_{k}^{-1} \hat{\mathbf{n}}_{k}$,

allowing one to iteratively obtain a solution for the maximum likelihood maps of each Stokes parameter.

\subsection{Sum and difference time streams}

An alternate approach to signal and noise estimation involves operations on the sum and difference of the calibrated time streams from bolometers within a PSB pair. This has the numerical advantage of isolating the temperature and polarization terms in the numerical inversion of Eq. (23). This approach takes full advantage of the high degree of common mode rejection of the PSB design, which is illustrated in Fig. 7. The advantages of this approach, which will be discussed in more detail in Sect. 3.6, are obtained at the cost of suboptimal noise weighting of the channels within a pair.

We may represent a sample, $i$, of a single detector as the linear combination of the sort,

$s_{i}=I+\gamma_{i}\left(Q \cos 2 \psi_{i}+U \sin 2 \psi_{i}\right)$.

Assuming that the channels are properly calibrated, the sum and difference of the signals from a PSB pair may be written as,

${ }^{+} s_{i} \equiv \frac{1}{2}\left(s_{1}+s_{2}\right)_{i}=I+\frac{1}{2}\left({ }^{+} \alpha_{i} Q+{ }^{+} \beta_{i} U\right)$

${ }^{-} s_{i} \equiv \frac{1}{2}\left(s_{1}-s_{2}\right)_{i}=\frac{1}{2}\left({ }^{-} \alpha_{i} Q+{ }^{-} \beta_{i} U\right)$, where we have defined the angular coefficients

$$
\begin{aligned}
{ }^{ \pm} \alpha_{i} & =\gamma_{1} \cos 2 \psi_{1 i} \pm \gamma_{2} \cos 2 \psi_{2 i} \\
{ }^{ \pm} \beta_{i} & =\gamma_{1} \sin 2 \psi_{1 i} \pm \gamma_{2} \sin 2 \psi_{2 i}
\end{aligned}
$$

in terms of the independent variables, $\psi_{k i}$, where $k=\{1,2\}$ identifies the channel. Recall that for a PSB pair the angular separation of the channels is $\Delta \simeq 90 \pm 2^{\circ}$, however this treatment in no way requires that to be the case.

Following the prescription of Sect. 3.3, one generates linear combinations of the differenced data,

$\left(\begin{array}{c}{ }^{-} s_{i}{ }^{-} \alpha_{i} \\ { }^{-} s_{i}{ }^{-} \beta_{i}\end{array}\right)=\frac{1}{2}\left(\begin{array}{cc}-\alpha_{i}^{2} & -\alpha_{i}{ }^{-} \beta_{i} \\ { }^{-} \alpha_{i}^{-} \beta_{i} & { }^{-} \beta_{i}^{2}\end{array}\right)\left(\begin{array}{c}Q \\ U\end{array}\right)$.

As before, one builds up information about the $Q, U$ decorrelation matrix through the combination of channel pairs, as well as modulation of the angular coverage, $\psi$. In this regard we have

${ }^{-} \hat{n}_{p}=\sum_{j}^{N_{\text {pairs }}} w_{j} \sum_{i \in p}\left(\begin{array}{c}{ }^{-} n_{i}{ }^{-} \alpha_{i} \\ { }^{-} n_{i}{ }^{-} \beta_{i}\end{array}\right)$,

where the time-streams ${ }^{-} n_{i}$ represent the polarization subtracted difference data. The $2 \times 2$ decorrelation matrix is, therefore,

${ }^{-} \hat{M}_{p}=\frac{1}{2} \sum_{j}^{N_{\text {pars }}} w_{j} \sum_{i \in p}\left(\begin{array}{cc}-\alpha_{i}^{2} & -\alpha_{i}{ }^{-} \beta_{i} \\ -\alpha_{i}{ }^{-} \beta_{i} & { }^{-} \beta_{i}^{2}\end{array}\right)$

and we note that we are now using suboptimal weighting of the pairs to generate corrections to the polarization map. Note that, for $\Delta \simeq 90^{\circ}$, the quantities ${ }^{-} \alpha$ and ${ }^{-} \beta$ have opposite parity, so that when averaged over a large sampling of $\psi$, the off-diagonals of Eq. (32) are small. Once the corrections to $Q$ and $U$ are obtained, one may substitute them in the sum for ${ }^{+} s$ to solve self consistently for $I$.

\subsection{Polarized cross-linking}

The iterative map-making methods described in Sects. 3.4 and 3.5 result in a self-consistent estimate of the signal and noise from the data that is "optimal" in the least-squares sense. However, instrumental effects and the method of generating maps from the time ordered data can introduce correlations in and remove signal from the time domain data. These effects generically limit the fidelity of the recovered Stokes parameter maps $^{8}$.

In simulations using noise correlations to process the signal only time streams according to Eq. (16), these effects appear as a residual between the input and recovered Stokes parameter maps. The spatial morphology and amplitude of these residuals depend on the amount of cross-linking in the scan strategy, the degree of polarization modulation, as well as the method used to decorrelate the $I, Q$, and $U$ parameters from the time stream. While these residuals do not introduce a bias to the pseudo- $C_{\ell}$ estimates of the power spectra, they do contribute to the signal

\footnotetext{
8 Common examples of such instrumental effects include the impact of the AC coupling of detector outputs, variations in the noise spectra between detectors, scan synchronous noise, polynomial removal of atmospheric signals, and limited accuracy of estimates of the low frequency noise. These noise estimates are fundamentally sample variance limited by the finite period over which the noise can be considered to be stationary.
} 

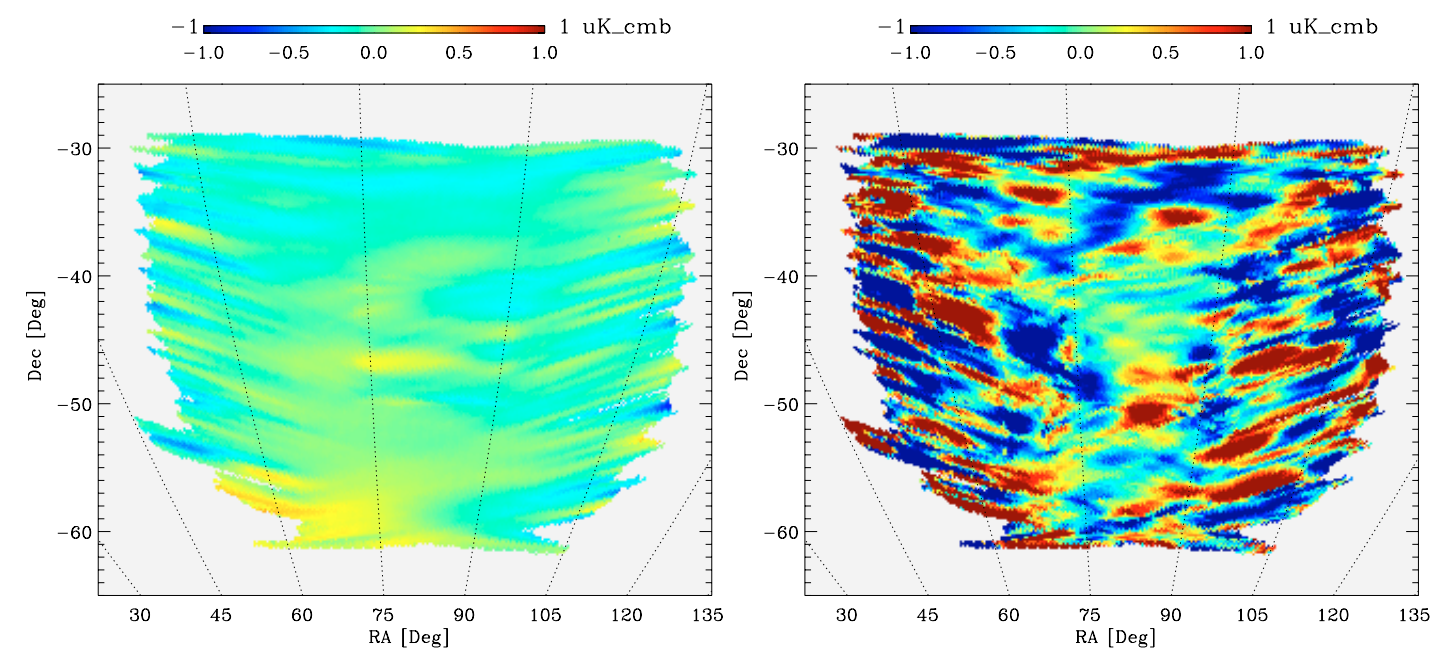

Fig. 8. A signal-only simulation, showing the residuals between the observed and input polarization (in this case, Stokes $Q$ ). The differencing method of Sect. 3.5 (left panel) is more robust to common mode effects than is the more general method of Sect. 3.4 (right panel), especially in regions where the crosslinking is poor. This is due primarily to the correlations that are introduced by the preconditioning of the TODs (essentially a highpass filter at $20 \mathrm{mHz}$ ) and the features in the noise kernel $N^{-1}$, which introduce path dependencies to the observed $I, Q$, and $U$ parameters.

covariance of the map, and therefore degrade the sensitivity of the Monte Carlo approach relative to optimal methods ${ }^{9}$.

The fidelity of the recovered Stokes parameter maps is an important consideration for the design of scanning polarimeters; the statistical depth of the survey determines the level at which these instrumental artifacts must be controlled. Unlike the noise contribution to the Stokes parameter maps, these artifacts do not integrate down, and can be mitigated only through improved cross-linking and modulation of the polarization.

To investigate these effects, we generate signal-only simulations based on the B03 observation strategy and the measured B03 noise power spectra. The B03 CMB data consist of a deep region and a shallow region, representing the extreme cases of possible observation strategies avaliable to Antarctic LDB payloads. The B03 scan crosses each pixel in the deep survey over many timescales and at many different orientations (due to sky rotation), while the pixels in the shallow survey are not well sampled.

Using the Healpix synfast facility (Górski et al. 2005), we generate a noise-free polarized CMB sky, pixelized at $3.4^{\prime}$ resolution, from a concordance $\Lambda \mathrm{CDM}$ model. We then simulate three polarization modulation schemes to compare with the nominal B03 modulation (i.e. sky rotation alone). Each time-domain simulation includes the nominal sky rotation in addition to that which would be achieved with a rotating half-wave plate. We model the following modulation schemes, which are representative of those proposed by balloon borne and terrestrial bolometric polarimeters (Oxley et al. 2004),

1. $22.5^{\circ}$ steps of the polarization angle each hour.

2. $22.5^{\circ}$ steps of the polarization angle at the end of every scan.

3. Continuous rotation of the polarization angle of each PSB at $350 \mathrm{mHz}^{10}$.

We observe the simulated sky with each of these polarization modulation schemes and create a noise-free time ordered data

9 The residuals contribute directly to the effective transfer function for the temperature and polarization spectra (the $\mathcal{F}_{\ell}$ discussed in Hivon et al. 2002 and Contaldi et al. 2005).

${ }^{10}$ We choose this modulation rate to be as fast as possible, given the BOOMERANG scan and sample rates. set, $s$, for each. We then solve for the signal part of the general least squares map using the B03 inverse noise filters, $N^{-1}$, according to Eq. (16):

$\tilde{m}=\left(A^{T} N^{-1} A\right)^{-1} A^{T} N^{-1} s$.

The noise kernels, $N^{-1}$, are smoothly truncated below $70 \mathrm{mHz}^{11}$. We decorrelate the Stokes $I, Q$, and $U$ parameters at $6.8^{\prime}$ resolution, using both the general $(3 \times 3)$ method and the PSB sum/difference $(2 \times 2)$ method, and compare the resulting polarization maps with the input sky. In the case of the former method, the residuals in the $Q$ and $U$ maps contain contributions from the finite resolution of the pixelization as well as the correlations introduced in the course of making maps from the time-ordered data. The difference time streams do not contain the relatively large unpolarized contribution, and therefore are far less susceptible to these pixelization effects.

Before considering the effects of the polarization modulation, we first investigate the benefits of exploiting the common mode rejection of the PSB pairs through the analysis of the difference time streams. We compare the residuals resulting from the application of the general method of Sect. 3.4 and from that of Sect. 3.5. In Fig. 8 we show the qualitative improvement in the fidelity of the reconstruction that results from the analysis of the difference time streams. It should be noted that, even for the general method, the sky rotation of the nominal B03 scan provides a degree of modulation that is sufficient to reduce the residuals to a level well below that of the instrumental noise in the B03 maps (Jones et al. 2006; Masi et al. 2005); the use of a waveplate in B03 would not have significantly improved the accuracy of the polarimetry. Furthermore, the direct difference method of Sect. 3.5 is less sensitive to the limited cross-linking of the nominal scan than is the general polarization decorrelation method of Sect. 3.4.

While the design of the PSBs is naturally suited to the sum/difference approach, scanning experiments sensitive to a

\footnotetext{
11 The lowest frequency that can be reliably recovered clearly affects the amplitude of the residuals, especially for the temperature fluctuations which have significant power on large scales. However, the relative benefits of the scanning strategies outlined above are generally insensitive to the exact value of the minimum frequency.
} 

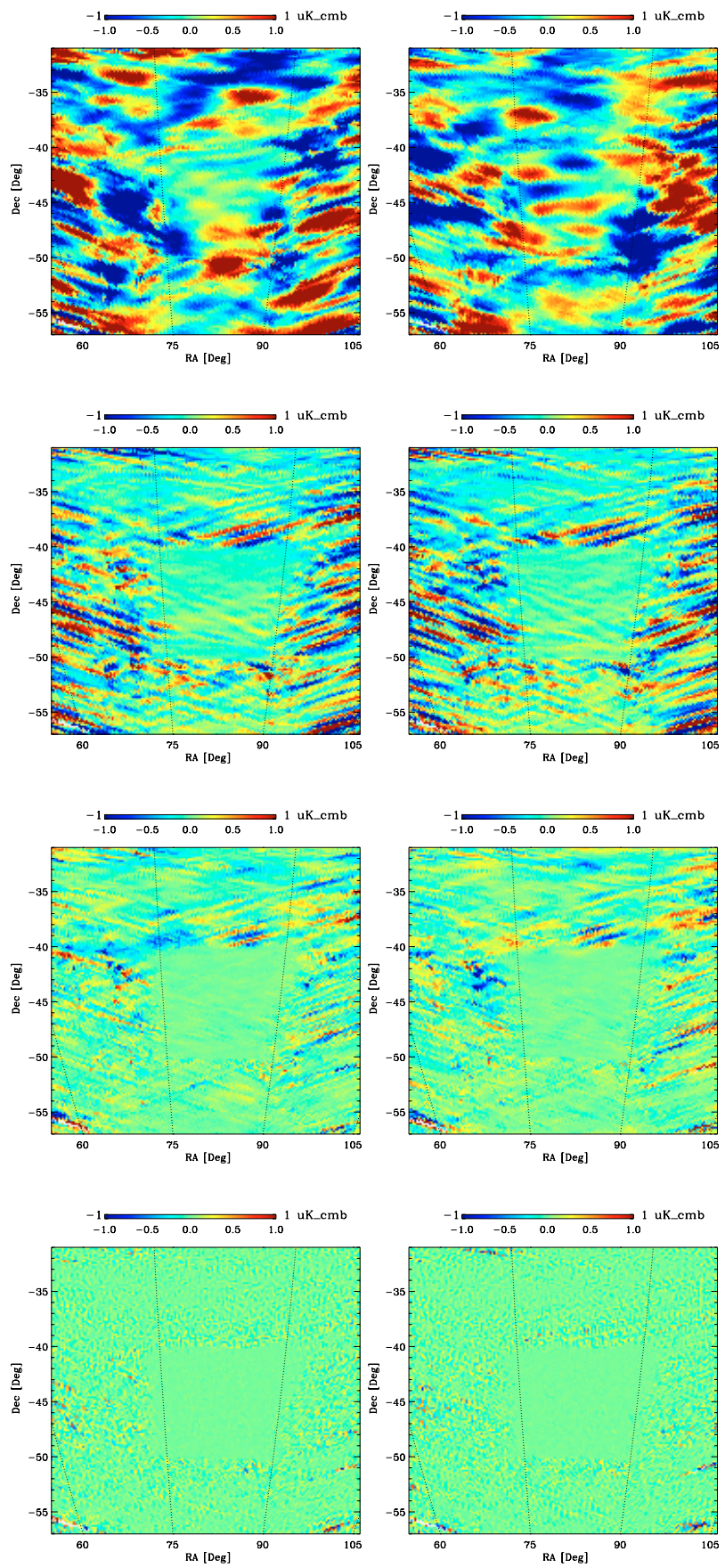

Fig. 9. The residual signal in the Stokes $Q$ (left column) and $U$ (right column) parameter maps generated using the general algorithm of Sect. 3.4, for increasing levels of polarization modulation. At top, the only modulation is that provided by sky rotation. The middle two sets are the residuals obtained when stepping the half waveplate by $22.5^{\circ}(Q \rightarrow U)$ each hour and at the end of each azimuth scan, respectively. The bottom row shows the fidelity achieved with a waveplate spinning continuously at $350 \mathrm{mHz}$. The remaining residuals are dominated by pixelization effects.

single polarization (such as EBEX Oxley et al. 2004 and SPIDER Netterfield et al. 2006) are not able to exploit the common mode rejection that is intrinsic to the design of the PSBs. A scheme for polarization modulation is therefore a highly desirable feature in singly polarized systems.

In order to illustrate the effect of the polarized cross-linking on the fidelity of the reconstructed signal, we show in Fig. 9

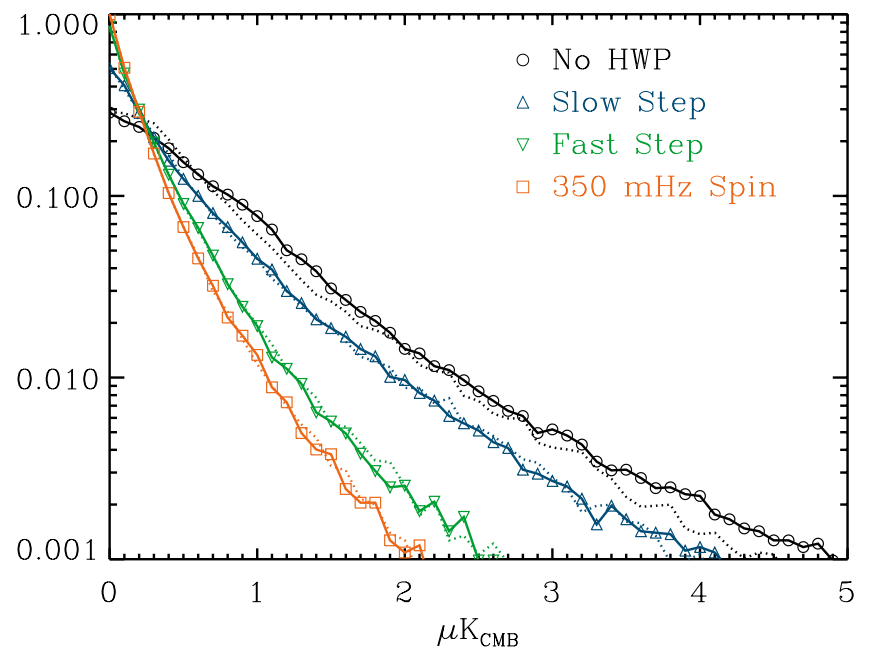

Fig. 10. Histograms of the Stokes $Q / U$ residuals, using 6.8' pixels, for various modulation schemes. Figure 9 shows that the errors are largest on large scales. The residuals in the last row are dominated by pixelization effects.

the residuals that result, for a particular CMB realization, from the general $(3 \times 3)$ approach of Sect. 3.4 for the nominal B03 scan, and for each of the three modulation schemes listed above. The fidelity of the signal reconstruction improves in proportion to the rate of modulation. The improved cross-linking randomizes the path dependencies of the observed Stokes parameters that are introduced by the processing of the time ordered data, namely Eq. (16).

As shown quantitatively in Fig. 10, the largest residuals in the $Q$ and $U$ maps (which, in the B03 example, occur on relatively large scales) can be significantly reduced by a modest degree of polarization modulation. Nevertheless, for the effects that have been included in this simulation, generating maps using the sum and difference time streams of the PSBs is nearly as effective at minimizing the residuals in the $Q$ and $U$ maps as the use of a half wave plate. It is important to note that beam asymmetries, instrumental polarization, pointing errors, and calibration uncertainties are examples of effects that are not included in these simulations. Each of these effects are mitigated by the use of an idealized modulation scheme, but not by the sum/difference method of Sect. 3.5.

\section{Summary and conclusions}

We have described in detail the design and performance of the Polarization Sensitive Bolometers (PSBs) which have enabled the first generation of successful bolometric CMB polarimeters. This discussion outlines the instrument parameters which must be characterized to accurately decorrelate the Stokes $I, Q$ and $U$ parameters from the time ordered data of a PSB. The design of the PSBs provides a high degree of common mode rejection that can be exploited in the analysis to minimize susceptibility to various instrumental effects that can potentially limit the fidelity of the recovered polarization. Simulations of PSB data including realistic instrumental effects illustrate the benefits of analyzing difference time streams, as well as various practical schemes for modulating the polarization signal.

Acknowledgements. The authors would like to thank Eric Hivon for many helpful discussions. The bolometers used by BoomERANG, QUaD, the Robinson telescope, and Planck HFI were fabricated at the Micro-Devices Laboratory at JPL (Jamie Bock and Anthony Turner). BooMERANG is an international collaboration between Caltech, JPL, IPAC, LBNL, U.C. Berkeley, Case Western Reserve 
University, University of Toronto, CITA, Cardiff University, Imperial College, Università di Roma, INGV, INFN, and the Institut d'Astrophysique.

\section{Appendix A: Noise in bolometric receivers}

\section{General noise properties}

Noise in bolometric receivers originates from several independent sources, including contributions from the readout electronics, the detector, and the intrinsic fluctuations in the optical background power. These noise sources are independent processes and their contributions add in quadrature to the total noise of the system.

The contribution of each of these components to the time ordered data are filtered by the transfer function of the one or both of the detector and the readout electronics ${ }^{12}$. The voltage noise (that is, the Johnson, JFET/amplifier noise, and the product of current noise with the series impedance) of the system, $n_{v}$, is filtered only by the transfer function of the readout, $\widetilde{Z}(f)$. The photon noise, $n_{\gamma}$, and phonon noise, $n_{G}$, are filtered not only by the bolometer voltage responsivity, $\widetilde{S}(f)$, but also by the readout. The bolometer transfer function, $\widetilde{S}$, is typically that of a single low pass filter, or a cascade of two such filters. The details of the readout transfer function, $\widetilde{Z}$, vary by application but always include an anti-aliasing filter that strongly attenuates frequencies well below the Nyquist frequency of the analog-to-digital converter $(A D C)^{13}$. The raw data are composed of the signal from the sky, $s$, and the various noise contributions, convolved with the bolometer and readout transfer functions,

$d=Z \otimes\left[S \otimes\left(s+n_{\gamma}+n_{G}\right)+n_{v}\right]$.

One of the first stages of analysis involves the deconvolution, and then the de-glitching, of this raw detector time stream. The deconvolution is normally accomplished in the Fourier domain by dividing the product of the bolometer and electronics transfer functions, $\widetilde{Z^{\prime}}=\widetilde{Z} \widetilde{S}$, from the raw data, $d$. While this results in a time stream that is characterized by a signal component with a uniform calibration in the frequency domain, the contribution of the voltage noise is biased according to the detector transfer function, $\widetilde{n}_{v} \rightarrow \widetilde{n}_{v} / \widetilde{S}$. Because the bolometer transfer function has the form of a low-pass filter, the deconvolved time stream generally exhibits " $f$-noise" in proportion to the time constant of the bolometer and the amplitude of the voltage noise component.

Figure 7 is an example of the power spectrum of a time stream prior to deconvolving the system transfer function. Figures 5 and 6 show the power spectrum of the deconvolved noise data. Accurate knowledge of the system transfer function is required to avoid the introduction of instrumental artefacts in the recovered signal. In the case of CMB studies, such an error will generally bias the power spectra that are derived from the maps (Jones et al. 2006).

Contemporary bolometric receivers, even those operating in the low background environment provided by balloon and orbital payloads, are designed to achieve background limited sensitivities. In these receivers photon noise represents a major, if not

\footnotetext{
12 For contemporary bolometric instruments like Planck HFI, the readout electronics include a cold JFET amplifier, ambient temperature amplifier/bandpass filters, and an anti-aliasing/data acquisition system. In practice, the contribution to the noise of everything except the JFET amplifiers and low noise preamplifier are negligible.

13 In some instances, the electronics are AC coupled, meaning that the low frequencies are strongly attenuated. The advent of low-cost, highresolution ADCs has made this feature less common.
}

dominant, contribution to the total noise in the system. In the following section we examine aspects of this photon noise contribution, $n_{\gamma}$, including a derivation of the (low level) noise correlations expected between detectors in a PSB pair resulting from fundamental properties of statistical fluctuations in the thermal background radiation.

\section{Photon noise}

A fundamental limitation to the sensitivity of any receiver (bandgap, coherent, or bolometric) derives from the intrinsic temporal fluctuations in the optical, often thermal, background radiation. The noise properties of thermal background radiation, or photon noise, differ greatly between radio, sub-millimeter, infrared, and optical instrumentation due to their vastly different operational regimes of photon occupation number. Photons satisfy Bose-Einstein statistics, and therefore the occupation of a mode of frequency $v$ is

$$
n(v, T)=\frac{1}{\mathrm{e}^{h v / k T}-1}
$$

for a thermal background of temperature, $T$.

The ratio $k / h=20.8[\mathrm{GHz} / \mathrm{K}]$ sets, for a given background temperature, the frequency for which average occupancy is above or below unity. At radio wavelengths astronomical instruments typically enjoy background levels of order $10 \mathrm{~K}$, with the minimum background limited by the CMB monopole at $2.728 \mathrm{~K}$. At higher frequencies, atmospheric loading and thermal emission from the instrument tend to dominate the background, and are typically $\sim 30-100 \mathrm{~K}$ for terrestrial telescopes. Therefore, instruments operating at frequencies above $\sim 100 \mathrm{GHz}$ have occupation numbers of order unity, while receivers at lower frequencies tend to have very large occupation numbers, $n \simeq k T / h v$. In the low $n$ regime, photons can be thought of as arriving at the detector sporadically. The photon noise in high frequency $(\gtrsim 100 \mathrm{GHz}$ ) instruments with low backgrounds can therefore be expected to largely satisfy Poisson statistics, where one expects fluctuations on the mean to scale roughly as $\sqrt{N}$.

Hanbury Brown and Twiss were the first to complete a rigorous analysis of noise correlations in photons Hanbury Brown \& Twiss (1956a,b, 1957a,b). The topic has been continually revisited in the fifty years since the first published work, and is still relatively un-advertised among many instrumentalists and observers alike. Therefore, we go through the analysis in detail.

Following Zmuidzinas (2003), we can write the covariance matrix describing detector outputs in all generality

$\sigma_{i j}^{2}=\frac{1}{\tau} \int \mathrm{d} v B_{i j}\left(B_{j i}+\delta_{i j}\right)$

where we define the power coupling matrix

$B_{i j} \equiv h v \sum_{k} S_{i k} S_{j k}^{*} n_{k}+C_{i j}$

and the internal noise term,

$C_{i j} \equiv\left(\mathbf{I}-\mathbf{S} \mathbf{S}^{\dagger}\right)_{i j} \frac{h v}{2} \frac{\mathrm{e}^{x}+1}{\mathrm{e}^{x}-1}$

Here, $S_{i j}$ is the standard scattering matrix, which couples an output amplitude, $a_{i}$, to the inputs, $b_{i}$, at each port of a network, as in Fig. A.1. The scattering matrix is defined by this relationship, $a_{i}=\sum_{j} S_{i j} b_{j}$. In Eq. (A.5), $x \equiv h v / k T_{S}$, where $T_{S}$ is the thermodynamic temperature of the system $S$, and the $n_{k}$ in Eq. (A.4) are 


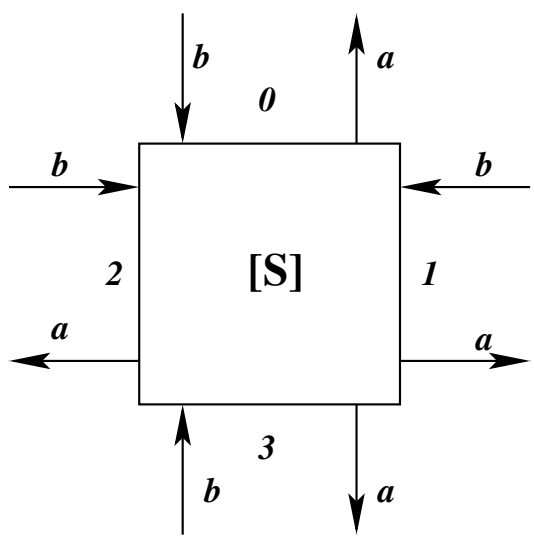

Fig. A.1. The scattering matrix of a four port network. A polarization sensitive bolometer can be modeled by such a network.

the occupation numbers of the modes at port $k$. We take ports 0 and 1 to label the two input polarization states, and let ports 2 and 3 label the two bolometers in a PSB pair. For simplicity, we assume that $n_{2}=n_{3}=0$ (i.e., the detectors are extremely cold with respect to the background), and that the input populations $n_{0}=n_{1}=n\left(v, T_{\text {load }}\right)$ imply no net polarization in the background.

The internal noise term, $C_{i j}$ arises as a result of losses in the system. Any mechanism causing loss implies a thermal noise contribution, $c_{i}$, to the outgoing signals

$a_{i}=\Sigma_{j} S_{i j} b_{j}+c_{i}$

which depends on the temperature of the lossy component.

In an ideal lossless network, the system's thermal noise term will vanish since $S$ is unitary, $\left(I-S S^{\dagger}\right)_{i j}=0$. In this case, the only nonzero terms in the scattering matrix are $S_{20}=S_{31}=1$. Since the only nonzero populations are $n_{0,1}=n$, the covariance matrix contains only terms with $B_{20}=B_{31}=n \cdot h v$. Under this assumption the detectors' noise is uncorrelated, and the autocorrelations satisfy

$\sigma_{i i}^{2}=\frac{(h v)^{2}}{\tau} \int \mathrm{d} v n(n+1)$.

Therefore the $1 \sigma$ uncertainty in the incident power due to intrinsic background fluctuations is

$\sigma_{\text {photon }}=\frac{h v}{\eta} \sqrt{\frac{\Delta v}{\tau}} \sqrt{\eta n(\eta n+1)}$.

Note that in the above we have explicitly included the optical efficiency, $\eta \equiv\left|S_{20}\right|^{2}=\left|S_{31}\right|^{2}$. This result differs from the familiar Dicke radiometer equation describing coherent receivers,

$\sigma_{\text {photon }}=\frac{h v}{\eta} \sqrt{\frac{\Delta v}{\tau}}(\eta n+1)$.

While Eq. (A.8) is the limiting form of Eq. (A.7) for large occupation number $n$, it is instructive to derive Eq. (A.8) from Eq. (A.3).

\section{Example 1: The Dicke radiometer equation}

The scattering matrix for an idealized coherent receiver with perfect isolation contains a single nonzero term, $\left|S_{10}\right|^{2}=G$, where $G$ is the gain of the system. An amplifier can be thought of as a population characterized by an inverted distribution of energy levels, such as that found in a maser or laser. Such systems are conveniently described in terms of a negative temperature. As $T \rightarrow{ }^{-} 0$, the sign of the $C_{i j}$ from Eq. (A.5) is reversed. The only nonzero element of $\mathbf{C}$ is $C_{11}=G-1$, and therefore $B_{11}=G n+G-1$. Application of Eq. (A.3) gives

$$
\begin{aligned}
\sigma_{11}^{2} & =\frac{(h v)^{2}}{\tau} \int \mathrm{d} v G^{2}\left[n+1-(G)^{-1}\right][n+1] \\
& =\Delta v \frac{(h v)^{2}}{\tau} G^{2}\left[(n+1)^{2}-\frac{(n+1)}{G}\right] .
\end{aligned}
$$

In the limit that $G$ is significantly larger than unity, the second term becomes negligible. Referencing the noise to the input, we recover the (lossless) Dicke radiometer equation, Eq. (A.8),

$$
\sigma_{11}=h v \sqrt{\frac{\Delta v}{\tau}}(n+1) .
$$

\section{Example 2: Polarization sensitive bolometers}

A dual polarized, single-moded receiver (coherent or bolometric) is completely described by a four port network. Polarization sensitive bolometers and coherent receivers using orthogonal mode transducers (OMTs) are two examples of such systems. We now derive the photon noise properties of a PSB pair.

The action of the network, $S$, is that of an imperfect polarized beam splitter, with two inputs and two detectors. The PSBs (two of the four ports, labeled say, as numbers 2 and 3) are assumed to be at cryogenic temperatures, and therefore contribute negligibly to the photon occupation number. Therefore, the entries in the scattering matrix relevant to the observed photon noise are limited to the lower left quadrant, namely $S_{20}=\gamma, S_{31}=$ $\gamma^{\prime}, S_{21}=\delta$, and $S_{30}=\delta^{\prime}$. Here the parameters $\gamma$ and $\delta$ describe the efficiency of transmission of the copolar amplitude and the crosspolar amplitude, respectively, and in practice $\gamma \gg \delta$.

Only the lower right quadrant of $S S^{\dagger}$ is nonzero,

$\left(S S^{\dagger}\right)_{22}=\gamma^{2}+\delta \delta^{\prime} \quad\left(S S^{\dagger}\right)_{23}=\delta\left(\gamma+\gamma^{\prime}\right)$ $\left(S S^{\dagger}\right)_{32}=\delta^{\prime}\left(\gamma+\gamma^{\prime}\right)\left(S S^{\dagger}\right)_{33}=\gamma^{\prime 2}+\delta \delta^{\prime}$.

The power coupling terms, $B_{i j}$, of interest are given by

$$
\begin{aligned}
B_{22} & =\left(\left|S_{20}\right|^{2} n_{0}+\left|S_{21}\right|^{2} n_{1}\right) h v+C_{22} \\
& =\left(\gamma^{2} n_{0}+\delta^{2} n_{1}+\left[1-\left(\gamma^{2}+\delta \delta^{\prime}\right)\right] n_{c}\right) h v \\
B_{23} & =\left(S_{20} S_{30} n_{0}+S_{21} S_{31} n_{1}\right) h v+C_{22} \\
& =\left(\gamma \delta^{\prime} n_{0}+\gamma^{\prime} \delta n_{1}-\delta\left(\gamma+\gamma^{\prime}\right) n_{c}\right) h v
\end{aligned}
$$

where we have written the thermal contribution of the network $n_{c} \equiv \frac{1}{2} \frac{\mathrm{e}^{x}+1}{\mathrm{e}^{x}-1}$

In the case of PSBs, the source of the modal coupling are the detectors themselves and/or the optics. In the case of the detectors, they are extremely cold compared to the background. For BOOMERANG, the reimaging optics and filters are also cooled, and have low emissivity. Therefore, we assume the thermal noise contribution of the network, $n_{c}$, is very small compared to the background populations, $n_{i}$. Furthermore, we assume that the background is isotropic, i.e., $n_{0}=n_{1}=n$. The covariance of the photon noise is then fully described by

$$
\begin{aligned}
\sigma_{i i}^{2} & =(h v)^{2} \frac{\Delta v}{\tau}\left[\left(\gamma^{2}+\delta^{2}\right)^{2} n^{2}+\left(\gamma^{2}+\delta^{2}\right) n\right] \\
\sigma_{i j}^{2} & =(h v)^{2} \frac{\Delta v}{\tau}\left[(2 \gamma \delta)^{2} n^{2}\right] .
\end{aligned}
$$


The autocorrelation, Eq. (A.16), contains terms proportional to both $n^{2}$ and $n$. The former is commonly referred to as the Bose contribution, or as a "photon bunching" term. Equation (A.17) shows that correlations between devices are proportional only to the Bose term, implying that PSBs operating under higher background loading conditions will exhibit a higher proportion of correlated noise than the same instrument operating in a lower background. For an idealized system, in which the polarization leakage $\delta$ is zero, the covariance between detectors vanishes since the two linear polarization states are statistically independent of one another.

In practice, we estimate the total optical background power, $Q$, arising from the $\mathrm{CMB}$, atmosphere, the telescope, and emission from within the cryostat. For simplicity, this optical background is treated as having originated from a single thermal source at an effective temperature $T_{\mathrm{RJ}}=Q / \eta k_{B} \Delta v$. The noise equivalent power from the background fluctuations is then given by Eq. (A.16),

$\mathrm{NEP}_{\text {photon }}^{2} \simeq 2 h v Q\left(1+\eta n\left(T_{\mathrm{RJ}}\right)\right)$.

This is, of course, only approximate as we do not treat the background sources independently. It is often the case, however, that a single thermal source contributes the majority of the background optical power.

\section{Appendix B: The Jacobi method}

We outline the application of the Jacobi method to the problem of mapmaking from scanning experiments, much of which can be generalized to other iterative algorithms such as the method of preconditioned conjugate gradients. We loosely follow the more complete discussions of the topic which can be found, for example, in Barrett et al. (1994); Acton (1990); Young (1971), and Press et al. (1997).

The Jacobi method is a robust numerical method of solving a set of linear equations, $\mathbf{A x}=\mathbf{b}$, for which the matrix $A$ is (or can be arranged to be) diagonally dominant. The great strength of the Jacobi method is that, subject to this requirement, it is guaranteed to converge although it may do so relatively slowly. Given a trial solution, one may estimate a new solution without inverting the matrix A simply by solving for each component, $x_{i}^{k+1}$, given an estimate of the values $\left\{x_{i}^{k}\right\}$,

$x_{i}^{k+1}=A_{i i}^{-1}\left(b_{i}-\sum_{j \neq i} A_{i j} x_{j}^{k}\right)$.

It is often convenient, and advantageous from a numerical point of view, to write the above in terms of a correction to the previous iteration,

$x_{i}^{k+1}=x_{i}^{k}+\delta x_{i}^{k+1}$

where

$\delta x_{i}^{k+1} \equiv \eta A_{i i}^{-1}\left(b_{i}-\sum_{j} A_{i j} x_{j}^{k}\right)$.

Here we have inserted a convergence parameter $\eta \lesssim 1$, which may be tuned to aid the convergence of the algorithm. In the limit that $\mathbf{A}$ is diagonal, the optimal value is $\eta=1$. Generally speaking, the larger the off-diagonal terms become, the lower the optimal value of $\eta$. Clearly the diagonals of $\mathbf{A}$ must not be near zero. Furthermore, as can be seen from Eq. (B.1), the solution will diverge if the absolute value of the sum of the off-diagonals is greater than the diagonal element of each row.

As an example, consider the following linear system:

$\left(\begin{array}{ccc}5 & -2 & 1 \\ 5 & -7 & 1 \\ -2 & 1 & 6\end{array}\right) \mathbf{x}=\left(\begin{array}{c}-1 \\ 0 \\ 1\end{array}\right)$

Setting $\eta=1$, and using the above procedure results in the following sequence of solutions:

$$
\begin{aligned}
x_{0} & =(0.000,0.000,0.000) \\
x_{1} & =(-0.200,0.000,0.167) \\
& \vdots \\
x_{5} & =(-0.291,-0.187,0.102) \\
& \vdots \\
x_{\infty} & =(-0.300,-0.200,0.100) .
\end{aligned}
$$

This example converges to twelve significant digits after 40 iterations, largely independent of $\mathbf{x}_{0}$, the trial solution. The rate of convergence does not scale strongly with the array size, so the solution is an efficient way of solving large systems of equations.

A minor modification to the above procedure results in the Gauss-Seidel algorithm, for which the estimate for each value $x_{i}^{k+1}$ incorporates the most recent estimate of the parameters $\left\{x_{j}^{k+1}\right\}_{j<i}$ instead of the set of values from the previous iteration. This procedure is less numerically robust, but tends to converge more rapidly than Jacobi iteration.

The application to Eq. (16) is clear; the Jacobi method provides a robust method of solving for the general least squares map. Equating Eqs. (16) and (B.1) we find the correspondence,

$$
\begin{aligned}
\mathbf{A} & \rightarrow \mathbf{C}_{N}^{-1} \equiv\left(\mathbf{A}^{T} \mathbf{N}^{-1} \mathbf{A}\right) \\
\mathbf{x} & \rightarrow \widetilde{\mathbf{m}} \\
\mathbf{b} & \rightarrow \mathbf{A}^{T} \mathbf{N}^{-1} \mathbf{d} .
\end{aligned}
$$

Recall that the matrix $\mathbf{A}$, which appears on the right hand side, is the pointing matrix and should not be confused with the general linear system described in Eq. (B.1). The algorithm we use for calculating the correction to an estimate of the least squares map, $\widetilde{\mathbf{m}}^{k}$, is simply

$$
\delta \widetilde{\mathbf{m}}^{k+1} \propto \operatorname{diag}\left(\mathbf{A}^{T} \mathbf{N}^{-1} \mathbf{A}\right)^{-1} \mathbf{A}^{T} \mathbf{N}^{-1}\left(\mathbf{d}-\mathbf{A} \widetilde{\mathbf{m}}^{k}\right) .
$$

In practice, one can simultaneously solve for the noise covariance matrix of the data, $\mathbf{N}$. This typically results in slightly slower convergence of the algorithm than when using a fixed noise estimate.

\section{References}

Acton, F. S. 1990, Numerical Methods that Work, 2nd edn. (Washington, DC: Mathematical Association of America)

Amblard, A., \& Hamilton, J.-C. 2004, A\&A, 417, 1189

Barkats, D., Bischoff, C., Farese, P., et al. 2004, [arXiv: astro-ph/0409380]

Barkats, D., Bischoff, C., Farese, P., et al. 2005, ApJS, 159,

Barrett, R., Berry, M., Chan, T. F., et al. 1994, Templates for the Solution of Linear Systems: Building Blocks for Iterative Methods, 2nd Edition (Philadelphia, PA: SIAM)

Beeman, J. 2001, http: //www.haller-beeman.com

Born, M., \& Wolf, E. 1980, Principals of Optics, sixth edition (Pergammon) Borrill, J. 1999, Phys. Rev. D, 59

Bowden, M., Taylor, A. N., Ganga, K. M., et al. 2004, in Proc. SPIE, 5489, 84, ed. J. M. Oschmann, Jr.

Contaldi, C. R., Bond, J. R., Crill, B. P., et al. 2005, in preparation 
Doré, O., Teyssier, R., Bouchet, F. R., Vibert, D., \& Prunet, S. 2001, A\&A, 374, 358

Górski, K. M., Hivon, E., Banday, A. J., et al. 2005, ApJ, 622, 759

Gaier, T., Lawrence, C. R., Seiffert, M. D., et al. 2003, New Astron. Rev., 47, 1167

Hamaker, J. P., \& Bregman, J. D. 1996, A\&A, 117, 161

Hanbury Brown, R., \& Twiss, R. Q. 1956a, Nature, 177, 27

Hanbury Brown, R., \& Twiss, R. Q. 1956b, Nature, 178, 1447

Hanbury Brown, R., \& Twiss, R. Q. 1957a, Proc. Roy. Soc. London., Ser. A, 242, 300

Hanbury Brown, R., \& Twiss, R. Q. 1957b, Nature, 179, 1128

Hecht, E. 1998, Optics, third edition (Reading, MA: Addison Wesley Longman, Inc.)

Hinshaw, G., Spergel, D. N., Verde, L., et al. 2003, Ap\&SS, 148, 135

Hivon, E., Górski, K. M., Netterfield, C. B., et al. 2002, ApJ, 567, 2

Jarosik, N., Barnes, C., Greason, M. R., et al. 2006, ArXiv Astrophysics e-prints

Jarosik, N., Bennett, C. L., Halpern, M., et al. 2003, ApJS, 145, 413

Jones, R. 1941a, J. Opt. Soc. Am., 31, 448

Jones, R. 1941b, J. Opt. Soc. Am., 31, 500

Jones, R. 1942, J. Opt. Soc. Am., 32, 446

Jones, W. C., Ade, P., Bock, J., et al. 2006, ApJ, accepted [arXiv: astro-ph/0507494]

Jones, W. C., Bhatia, R. S., Bock, J. J., \& Lange, A. E. 2003, Proc. SPIE Int. Soc. Opt. Eng., 4855

Kogut, A., Spergel, D. N., Barnes, C., et al. 2003, Ap\&SS, 148, 161

Lamarre, J. M., Puget, J. L., Bouchet, F., et al. 2003, New Astron. Rev., 47, 1017

Leitch, E. M., Kovac, J. M., Halverson, N. W., et al. 2004, ApJ, submitted [arXiv:astro-ph/0409357]

Leitch, E. M., Kovac, J. M., Pryke, C., et al. 2002, Nature, 420, 763

Ludwig, A. 1973, IEEE Trans. Ant. Prop., AP-21, 116

MacTavish, C. J., Ade, P. A. R., Bock, J. J., et al. 2005, ApJ, accepted [arXiv: astro-ph/0507503]

Masi, S., Ade, P., Bock, J., et al. 2005, ArXiv Astrophysics e-prints
Montroy, T. E., Ade, P. A. R., Bock, J. J., et al. 2006, ApJ, accepted [arXiv: astro-ph/0507514]

Mueller, H. 1948, J. Opt. Soc. Am., 38, 661

Netterfield, C. B., Ade, P. A. R., Bock, J. J., et al. 2002, ApJ, 571, 604

Netterfield, C. B., et al. 2006, Proceedings of the 2005 UCI Cosmology Conference

O’Dell, C. W., Keating, B. G., de Oliveira-Costa, A., Tegmark, M., \& Timbie, P. T. 2003, Phys. Rev. D, 68, 042002

Olver, A. D., Clarricoats, P. J. B., Kishk, A. A., \& Shafai, L. 1994, Microwave horns and feeds, IEEE Electromagnetic Waves Series, 39

Oxley, P., Ade, P. A., Baccigalupi, C., et al. 2004, Infrared Spaceborne Remote Sensing XII, 5543, 320

Page, L., Nolta, M. R., Barnes, C., et al. 2003, ApJS, 148, 233

Piacentini, F., Ade, P., Bock, J., et al. 2006, ApJ, accepted [arXiv: astro-ph/0507507]

Press, W. H., Teukolsky, S. A., Vetterling, W. T., \& Flannery, B. P. 1997, Numerical Recipes, 2nd edn. (Cambridge: Cambridge University Press)

Prunet, S., Ade, P. A. R., Bock, J. J., et al. 2001, in Mining the Sky, 421

Readhead, A. C. S., Myers, S. T., Pearson, T. J., et al. 2004, Science, 306, 836

Ruhl, J. E., Ade, P. A. R., Bock, J. J., et al. 2003, ApJ, 599, 786 [arXiv: astro-ph/0212229]

Spiga, D., Battistelli, E., Boella, G., et al. 2002, New Astron., 7, 125 [arXiv:astro-ph/0202292]

Wright, E. L., Hinshaw, G., \& Bennett, C. L. 1996, ApJ, 458, L53

Yoon, K. W., Ade, P. A. R., Barkats, D., et al. 2006, in Proceedings of the SPIE, Vol. 6275, Millimeter and Submillimeter Detectors and Instrumentation for Astronomy III, ed. J. Zmuidzinas, W. S. Holland, S. Withington, \& W. D. Duncan, Bellingham, Washington [arXiv: astro-ph/0606278]

Young, D. 1971, Iterative Solutions of Large Linear Systems (New York: Academic Press)

Yun, M., et al. 2003, Proc. SPIE Int. Soc. Opt. Eng., 4855, 136

Zmuidzinas, J. 2003, Appl. Opt., 42, 4989 